\title{
Mineral chemistry of late Variscan gabbros from central Spain: constraints on crystallisation processes and nature of the parental magmas
}

\author{
D. Orejana ${ }^{1 *}$, E. Losantos ${ }^{2}$, C. Villaseca ${ }^{1,2}$, T.E. Jeffries ${ }^{3}$ \\ ${ }^{1}$ Department of Petrology and Geochemistry, Complutense University of Madrid, Madrid, Spain \\ ${ }^{2}$ Institute of Geosciences (UCM, CSIC). Madrid. Spain \\ ${ }^{3}$ Department of Mineralogy, Natural History Museum, London, United Kingdom \\ e-mail addresses:dorejana@ucm.es (D.O., *Corresponding author),e.losantos@csic.es (E.L.), granito@ucm.es (C.V.), \\ t.jeffries@nhm.ac.uk (T.E.J.)
}

Received: 19 May 2015 / Accepted: 7 July 2015 / Available online: 20 July 2015

\begin{abstract}
Major and trace element chemistry has been determined in the main minerals (olivine, orthopyroxene, clinopyroxene, plagioclase, amphibole, phlogopite) from the Talavera and La Solanilla gabbroic intrusions (Spanish Central System). The results show a heterogeneous mineral composition illustrative of the chemical evolution of calc-alkaline basic magmas during crystallization. The formation of incompatible element-rich interstitial minerals, such as amphibole, phlogopite and accessory phases (e.g., zircon, apatite), and the presence of zoning patterns in major minerals towards a more evolved composition, point to a progressive differentiation of the parental melt. The chemical variation depicted by trace elements usually incompatible with clinopyroxene (e.g., rare earth elements (REE), Th, Zr, Nb, Ti) implies co-precipitation with phases displaying a compatible behaviour with respect to these elements (e.g., apatite, zircon, amphibole). However, the very high LILE (large ion lithophile elements) and LREE (light REE) shown by clinopyroxene can not be explained exclusively by a closed-system in-situ crystallization process. Several features of the mineral chemistry (e.g. positive correlation of $(\mathrm{La} / \mathrm{Sm})_{\mathrm{N}}$ with La and $\mathrm{Na}$ in clinopyroxene and plagioclase, respectively) support the involvement of an external component (wall-rock assimilation or hybridization with an evolved melt). The petrography and mineral chemistry of the gabbros, in conjunction with bulk rock data from previous studies, favour a calc-alkaline affinity for the parental melts. Thus, metasomatism in the mantle sources related with this basic magmatism might be associated with recycling of crustal components during the Variscan orogeny.
\end{abstract}

Keywords: Late orogenic gabbros, late-stage crystallization, Spanish Central System, Variscan Orogeny, calc-alkaline magmas

\section{Resumen}

Se ha caracterizado la composición química de elementos mayoritarios y trazas en los minerales principales de las intrusiones gabroideas de Talavera y La Solanilla (Sistema Central Español). Los resultados muestran una composición mineral heterogénea que es ilustrativa de una evolución química de los magmas básicos calco-alcalinos durante la cristalización. La formación de minerales intersticiales con elevados contenidos en elementos incompatibles, como por ejemplo el anfíbol, la flogopita y algunos minerales accesorios (circón, apatito...), y la presencia de zonados en los minerales principales, con tendencia a desarrollar una composición más evolucionada hacia el borde, indican la diferenciación progresiva del fundido parental. La variación química de elementos traza normalmente incompatibles en el clinopiroxeno (e.g., REE, Th, Zr, Nb, Ti) implica la co-precipitación de fases con un comportamiento compatible con dichos elementos (e.g., apatito, circón, anfíbol). Sin embargo, las elevadas concentraciones de LILE y LREE del clinopiroxeno no se pueden explicar exclusivamente por un proceso de cristalización en un sistema cerrado. Varias características de la química mineral (e.g., correlación positiva de la relación (La/ $\mathrm{Sm})_{\mathrm{N}}$ con el contenido de La y $\mathrm{Na}$ en el clinopiroxeno y la plagioclasa, respectivamente) suponen la participación de un componente externo (asimilación de roca encajante o mezcla con un fundido más evolucionado). La petrografía y la química mineral de los gabros, junto con los datos de roca total de estudios previos, favorece una afinidad calco-alcalina para los fundidos parentales. Por lo tanto, el metasomatismo en las fuentes de manto asociadas con este magmatismo básico podría estar relacionado con el reciclaje de componentes corticales durante la orogenia Varisca. 


\section{Introduction}

During cooling of magmatic bodies, re-equilibration of interstitial melts with early-formed mineral assemblages is a key process which might influence the final bulk rock composition (e.g. Barnes, 1986). The relevance of late-magmatic processes in the geochemical evolution of magmas has been highlighted by many studies focused on mafic intrusions from oceanic (e.g. Ross and Elthon, 1997; Tribuzio et al., 1999b, 2000; Natland and Dick, 2001; Gao et al., 2007) and continental (e.g. Cawthorn et al., 1992; Bédard, 1994; Tribuzio et al., 1999a; Claeson and Meurer, 2004; Tiepolo and Tribuzio, 2005) environments. These studies have shown that in-situ crystallization of evolved interstitial melts directly controls the final mineral composition, and may result in enrichment of incompatible trace elements in the whole-rock composition. In accordance with these observations, a variable proportion of interstitial minerals, including clinopyroxene, $\mathrm{H}_{2} \mathrm{O}$-bearing phases (amphibole, phlogopite) and other accessory minerals (e.g. apatite, zircon, ilmenite, baddeleyite) are commonly formed between early-precipitated crystals (e.g. Tribuzio et al., 1999a, b, 2000; Tiepolo and Tribuzio, 2005; Borghini and Rampone, 2007). Late overgrowth of the liquidus phases may also occur (e.g. Barnes, 1986; Borghini and Rampone, 2007). Even though most of the above studies deal with cumulate rocks, the processes they discuss for the final stages of crystallization, may also operate during solidification of non-cumulate mafic rocks.

Another important aspect is the possible interaction between early crystallized minerals and a variable proportion of evolved interstitial liquid. The outcome of this process will be very different in a "closed system" crystallization process (e.g. Barnes, 1986; Bédard, 1994; Charlier et al., 2005; Bernstein, 2006) and if an external component, such as an evolved melt associated with wall rock assimilation or felsic magma hybridization, plays a major role (e.g. Tribuzio et al., 1999a; Tiepolo and Tribuzio, 2005; Renna et al., 2006). Mineral-melt interaction during late stage crystallization has been identified in rocks of variable geochemical affinity, including tholeiitic (Gillis, 1996; Tribuzio et al., 1999b, 2000; Coogan et al., 2001) and continental calc-alkaline magmas, the latter sometimes associated with post-collisional geodynamic settings (Monjoie et al., 2005; Tiepolo and Tribuzio, 2005; Renna et al., 2006). The nature of magmas formed during orogenic events is commonly unclear due to the involvement of several geochemical reservoirs (crust and mantle) and the leading role played by differentiation processes (e.g., fractional crystallisation and crustal contamination), which may obliterate the primary geochemical signature.

The determination of major and trace element mineral composition by microanalytical techniques (Electron Microprobe and Laser Ablation ICP-MS) in rock suites subjected to complex crystallization stories has proved as a main tool in deciphering the type of operating magmatic processes (e.g., Tribuzio et al., 1999b; Coogan et al., 2001; Claeson and Meurer, 2004; Bernstein, 2006; Borghini and Rampone, 2007). This approach allows the characterization of trace element mineral zoning and other micro-scale chemical heterogeneities.

In the present study we have analysed the major and trace element composition of the main rock-forming minerals from two gabbroic intrusions cropping out in the Spanish Central System (SCS) (Variscan Iberian Belt). These Variscan gabbros have been previously studied with special attention focused on the whole-rock chemistry (e.g. Orejana et al., 2009; Scarrow et al., 2009), the major element mineral data (Molina et al., 2009), and their zircon isotope (U-Pb, Hf) geochemistry (Villaseca et al., 2011). Different hypothesis have been proposed for their origin, advocating either primary calc-alkaline magmas (Orejana et al., 2009) or alkaline mantle-derived melts mixed with felsic magmas at the crust-mantle boundary (Scarrow et al., 2009). Our study represents the first attempt to determine the trace element mineral composition of SCS gabbroic rocks, especially regarding the chemical heterogeneity and zoning of interstitial phases. This kind of mineral data makes it possible to characerize late magmatic processes and help constrain the nature of the parental magmas.

\section{Geological Setting}

The Spanish Central System (SCS) batholith is mainly formed by more than 100 granitic intrusions (e.g. Bea et al., 1999; Villaseca and Herreros, 2000) emplaced within Neoproterozoic to Palaeozoic metaigneous and metasedimentary series, which represent the innermost continental region of the Iberian Variscan Belt (Fig. 1). Although these intrusions are predominantly peraluminous monzogranites, several series have been distinguished, including S-type peraluminous granites, I-type metaluminous to peraluminous plutons and transitional peraluminous granitoids (e.g. Recio et al., 1992; Pinarelli and Rottura, 1995; Villaseca et al., 1998; Villaseca and Herreros, 2000). Several models have been proposed to explain the origin of this huge batholith: a) hybridization between crustal melts and mantle-derived magmas (Pinarelli and Rottura, 1995; Moreno-Ventas et al., 1995); b) variable degrees of crustal assimilation by mantle derived magmas (Ugidos and Recio, 1993; Castro et al., 1999); and c) partial melting of essentially crustal sources, either from lower crustal derivation (Villaseca et al., 1998, 1999) or from midcrustal levels (Bea et al., 1999, 2003). Clear geochemical similarities between SCS granites and lower crustal xenoliths carried by the SCS Upper Permian alkaline dykes, suggest that the lower crust is the most likely crustal source for the formation of the SCS batholith (Villaseca et al., 1999, 2007). This is reinforced by coincidence in the $\mathrm{U}-\mathrm{Pb}$ zircon age ranges recorded in the SCS granites and the lower crustal xenoliths (Fernández-Suárez et al., 2006; Orejana et al., 2011).

Basic magmatism in the SCS batholith is represented by several series of fairly different geochemical affinity (calc- 


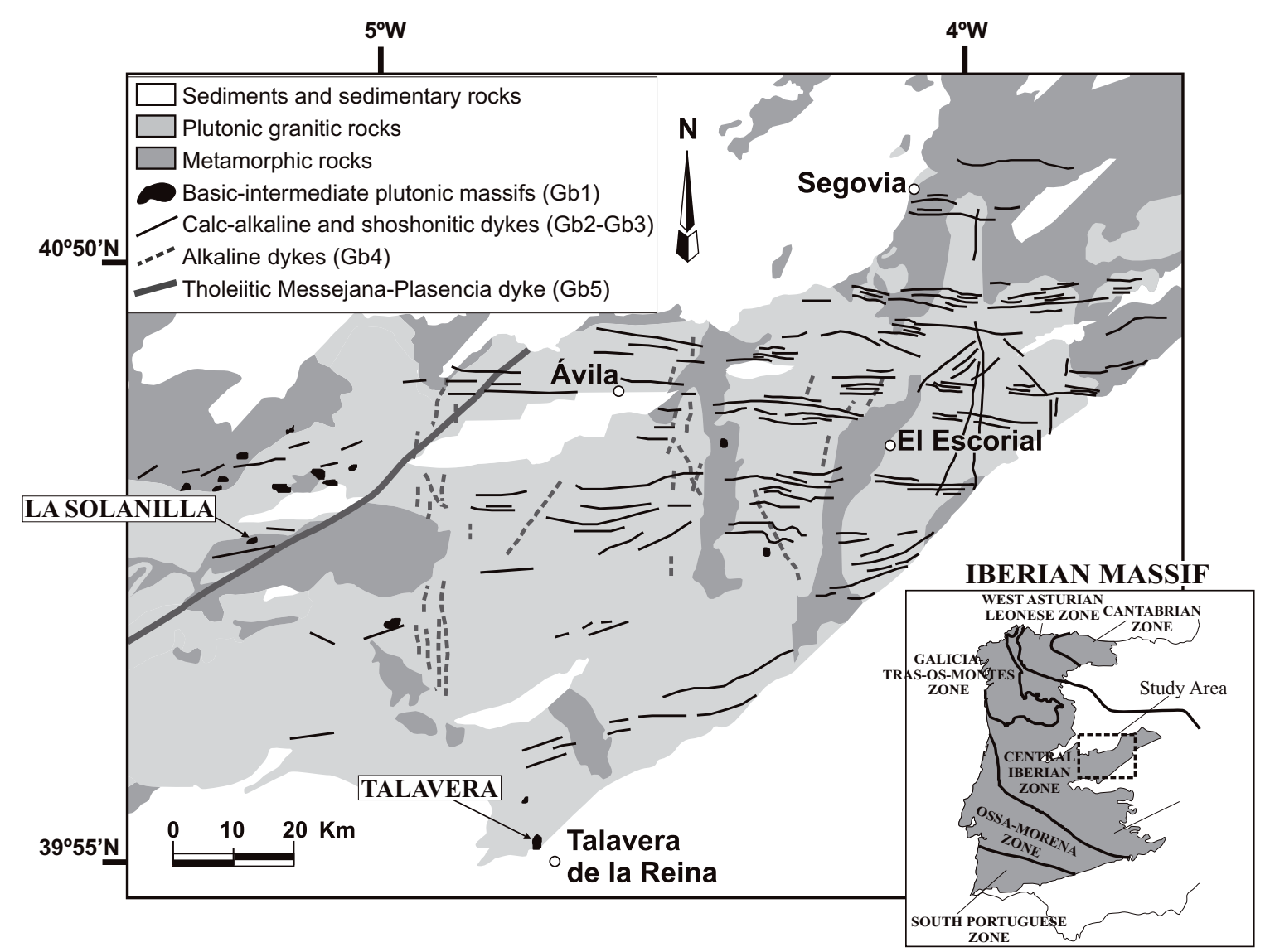

Fig. 1.- Geological map of the Spanish Central System showing the location of the studied Variscan gabbros (Talavera and La Solanilla), modified from Franco and García de Figuerola (1986), Franco and Sánchez García (1987), and Orejana et al. (2009).

alkaline, alkaline and tholeiitic) (Fig. 1), which intruded from Carboniferous to Jurassic (e.g. Villaseca et al., 2004). The alkaline and tholeiitic mafic rocks are post-Variscan dyke swarms associated with a rifting regime prior to the Atlantic Ocean opening (e.g. Cebriá et al., 2003; Orejana et al., 2008). The calc-alkaline gabbroic intrusions, which are mainly concentrated in the western SCS (Franco and García de Figuerola, 1986; Franco and Sánchez-García, 1987), are small (usually $<1 \mathrm{~km}$ in length) Variscan post-tectonic plutons with ages mainly in the range 305-300 Ma (Zeck et al., 2007; Villaseca et al., 2011). According to recent data, they are coeval with the main SCS granitoid intrusions ( 308-298 Ma; Zeck et al., 2007; Díaz-Alvarado et al., 2011; Orejana et al., 2012).

Recent studies have focused their attention on the nature and petrogenesis of the SCS mafic bodies (Molina et al., 2009, 2012; Orejana et al., 2009; Scarrow et al., 2009; Villaseca et al., 2011). These gabbros exhibit high incompatible trace element concentrations (LILE, LREE, $\mathrm{Pb}$ ), in conjunction with negative $\mathrm{Nb}-\mathrm{Ta}-\mathrm{Ti}$ anomalies, and a heterogeneous $\mathrm{Sr}-\mathrm{Nd}$ isotopic composition (e.g. Orejana et al., 2009; Scarrow et al., 2009). As a result, the most primitive gabbros have been interpreted as derived from melting of a heterogeneous subcontinental lithospheric mantle, metasomatised via crustal recycling (Orejana et al., 2009). However, other studies favour a mixing process in the deep crust involving alkaline mantle-derived magmas and crustal melts (e.g. Scarrow et al., 2009). In any case, subduction is not considered to be directly linked with the genesis of the gabbros, and melting would have been caused by adiabatic decompression after the collision ended (Orejana et al., 2009; Scarrow et al., 2009).

Additionally, hybridization of these basic magmas with crustal melts during transport and emplacement has been described by Moreno-Ventas et al. (1995), Scarrow et al. (2009) and Villaseca et al. (2011). Other studies on Iberian Variscan gabbros have also highlighted the existence of crustal contamination at shallow levels on the basis of whole-rock chemistry (e.g. Dias and Leterrier, 1994; Galán et al., 1996; López-Moro and López-Plaza, 2004). More recently, it has been observed that differences in initial $\varepsilon H f$ values between zircon crystals from several gabbroic bodies are in accordance with the heterogeneity of whole-rock $\mathrm{Sr}-\mathrm{Nd}$ isotopic data, and that within sample $\varepsilon \mathrm{Hf}$ variability also indicates that crustal assimilation and felsic magma hybridization occurred in some of these intrusions (Villaseca et al., 2011).

The two gabbroic bodies studied for the present study (Talavera and La Solanilla) are hosted by granitic and metamorphic rocks. Contact relations with the wall-rock can not be clearly observed in the field. Samples collected from different outcrops in the inner areas are fairly homogeneous in terms of bulk-rock (Orejana et al., 2009) and mineral composition. The main gabbro bodies do not show modal layering or textural variations. This homogeneity and their primitive character, make these 


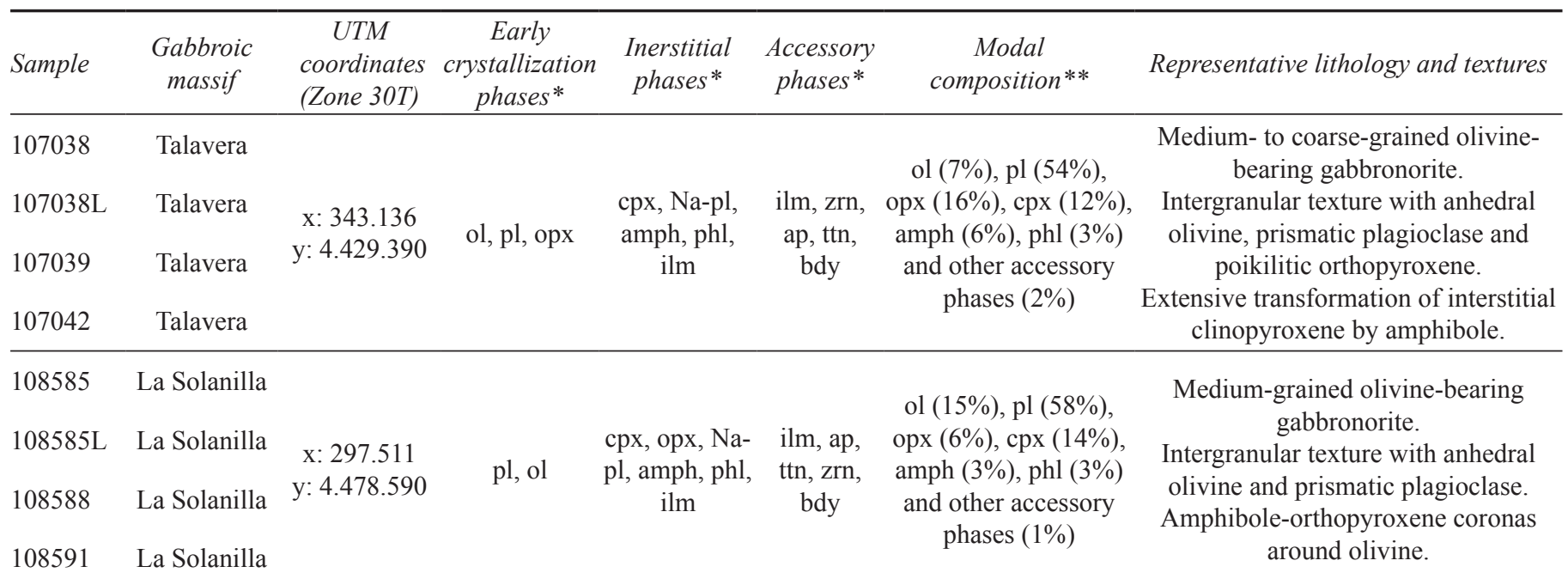

*ol: olivine, opx: orthopyroxene, cpx: clinopyroxene, pl: plagioclase, Na-pl: Na-rich interstitial plagioclase, amph: amphibole, phl: phlogopite, ilm: ilmenite, zrn: zircon, ap: apatite, ttn: titanite, bdy: baddeleyite.

**Modal composition has been estimated by point counting performed on thick sections $\mathrm{n}^{\circ} 107038 \mathrm{~L}$ and $108585 \mathrm{~L}$ (see the analytical methods for more details).

Table 1.- Summary of petrographic features of analysed SCS olivine-bearing gabbros

gabbros suitable for the study of the magmatic processes which operated during cooling of the parental basic magma.

\section{Analytical methods}

Eight samples were selected for the present study: four from the Talavera gabbro and four from the La Solanilla gabbro (Fig. 1; Table 1). Thin sections were used for petrographic observations and for a detailed determination of the mineral mode. The latter was done statistically considering the relative abundance of the main minerals within a virtual network of 1000-2000 points (depending on the sample surface) (see results in Table 1). Major element compositions of olivine, orthopyroxene, clinopyroxene, plagioclase, amphibole and phlogopite has been analysed directly on polished thin and thick $(130 \mu \mathrm{m})$ sections at the Centro Nacional de Microscopía Electrónica (Complutense University of Madrid) using a JEOL JXA-8900 M electron microprobe with four wavelength-dispersive spectrometers. A selection of representative analyses has been included in Table 2, but the whole dataset is available as a supplementary electronic material (Supp. eTable 1). Analytical conditions were an accelerating voltage of $15 \mathrm{kV}$ and an electron beam current of 20 $\mathrm{nA}$, with a beam diameter of $5 \mu \mathrm{m}$. Elements were counted for $10 \mathrm{~s}$ on the peak and $5 \mathrm{~s}$ on each background position. Natural and synthetic standards were employed. Corrections were made using an on-line ZAF method. Detection limits are $0.02 \mathrm{wt} \%$ for $\mathrm{Al}, \mathrm{Na}$ and $\mathrm{K} ; 0.03 \mathrm{wt} \%$ for $\mathrm{Ti}, \mathrm{Fe}, \mathrm{Mn}, \mathrm{Mg}$, $\mathrm{Ni}$ and $\mathrm{Cr}$, and $0.04 \mathrm{wt} \%$ for $\mathrm{Si}$.

We have determined in situ concentrations of 28 trace elements (REE, Ba, Rb, Sr, Th, U, Nb, Ta, Pb, Zr, Hf, Y, Sc, V, $\mathrm{Co}$ and $\mathrm{Cr}$ ) in olivine, plagioclase, orthopyroxene, clinopyroxene, amphibole and phlogopite on two polished thick sections $(130 \mu \mathrm{m})$ by laser ablation ICP-MS at the Natural History Museum of London, using a New Wave UP193FX gas laser source operating at $193 \mathrm{~nm}$ coupled to an Agilent $7500 \mathrm{CS}$ ICP-MS. A selection of representative analyses has been included in Table 3, but the whole dataset is available as supplementary electronic material (Supp. eTable 2). Laser pulse energy was ca $0.2 \mathrm{~mJ}$ per pulse, energy density ca 3.5 $\mathrm{J} / \mathrm{cm}^{2}$, and frequency was set at $10 \mathrm{~Hz}$. Laser ablated sample particles were carried in a flow of $\mathrm{He}$ gas to a high temperature $(10000 \mathrm{~K})$ Ar plasma. The diameter of the laser beam was about $50 \mu \mathrm{m}$. Twenty analyses were performed in each run, the first two and last two corresponding to the calibration standard NIST 612 (a synthetic silicate glass; Pearce et al., 1997). The remaining 16 analyses were done on the selected samples. The counting time for one analysis was typically $90 \mathrm{~s}$ (40 s measuring gas blank to establish the background and $50 \mathrm{~s}$ for the remainder of the analysis). Analyses were monitored to detect accessory phase inclusions. Data were subsequently processed offline using LAMTRACE software, a programme written by Simon Jackson (Geological Survey of Canada) for handling time-resolved LA-ICP-MS data. Each analysis was normalized to $\mathrm{Ca}$ or Si using concentrations determined by electron microprobe. Detection limits for each element were in the range of $0.01-0.06 \mathrm{ppm}$, except for $\mathrm{Sc}$ and $\mathrm{Cr}$ (0.11 and $0.73 \mathrm{ppm}$, respectively) and a precision of $5 \%$ relative standard deviation was assumed.

\section{Petrography}

The studied samples represent medium- to coarse-grained olivine-bearing gabbronorites with intergranular texture, being olivine and plagioclase the early magmatic minerals (Fig. $2 \mathrm{a}, \mathrm{b})$. Olivine appears as anhedral, often rounded grains, whereas plagioclase forms euhedral to subhedral prisms with apparent concentric zoning.

The Talavera sample shows large anhedral poikilitic orthopyroxene crystals, leading to a hypidiomorphic subophitic 


\begin{tabular}{|c|c|c|c|c|c|c|c|c|c|c|c|c|c|c|c|c|c|}
\hline \multirow{3}{*}{$\begin{array}{l}\text { Sample } \\
\text { Analysis }\end{array}$} & \multicolumn{3}{|c|}{ olivine } & \multicolumn{4}{|c|}{ orthopyroxene } & \multicolumn{10}{|c|}{ clinopyroxene } \\
\hline & \multirow{2}{*}{$\begin{array}{c}\text { Talavera } \\
107038 \mathrm{~L} \\
16 \mathrm{c}\end{array}$} & \multirow{2}{*}{\multicolumn{2}{|c|}{$\frac{\text { La Solanilla }}{108585 \mathrm{~L}}$}} & \multirow{2}{*}{\multicolumn{2}{|c|}{$\frac{\text { Talavera }}{107038 \mathrm{~L}}$}} & \multirow{2}{*}{\multicolumn{2}{|c|}{$\frac{\text { La Solanilla }}{108585 \mathrm{~L}}$}} & \multirow{2}{*}{\multicolumn{5}{|c|}{ Talavera }} & \multicolumn{5}{|c|}{ La Solanilla } \\
\hline & & & & & & & & & & & & & & & $08585 \mathrm{I}$ & & \\
\hline $\mathrm{SiO}_{2}$ & 36.67 & 37.26 & 37.06 & 52.70 & 53.16 & 52.70 & 53.38 & 51.44 & 52.78 & 52.33 & 51.91 & 52.62 & 50.87 & 52.04 & 50.91 & 50.84 & 51.56 \\
\hline $\mathrm{TiO}_{2}$ & 0.07 & 0.01 & 0.02 & 0.28 & 0.39 & 0.20 & 0.06 & 0.88 & 0.61 & 0.61 & 0.49 & 0.24 & 0.73 & 0.31 & 0.45 & 1.09 & 0.91 \\
\hline $\mathrm{NiO}$ & 0.04 & 0.03 & 0.08 & 0.02 & 0.10 & 0.00 & 0.02 & 0.01 & 0.01 & 0.00 & 0.00 & 0.01 & 0.07 & 0.02 & 0.00 & 0.00 & 0.11 \\
\hline $\mathrm{MnO}$ & 0.01 & 0.38 & 0.36 & 0.53 & 0.03 & 0.48 & 0.42 & 0.00 & 0.19 & 0.21 & 0.18 & 0.30 & 0.10 & 0.26 & 0.21 & 0.15 & 0.20 \\
\hline $\mathrm{MgO}$ & 33.52 & 31.78 & 33.26 & 24.97 & 26.90 & 23.65 & 25.43 & 16.51 & 17.40 & 15.35 & 16.71 & 15.14 & 16.46 & 17.18 & 18.90 & 14.80 & 15.22 \\
\hline $\mathrm{CaO}$ & .00 & 0.02 & 0.33 & 0.85 & 2.53 & 0.46 & 0.25 & 19.54 & 17.63 & 20.41 & 18.15 & 22.66 & 18.63 & 19.12 & 15.73 & 19.06 & 18.85 \\
\hline $\mathrm{Na}_{2} \mathrm{O}$ & 04 & 0.00 & 0.09 & 0.04 & 0.06 & 0.00 & 0.00 & 0.40 & 0.29 & 0.44 & 0.48 & 0.43 & 0.71 & 0.35 & 0.40 & 1.12 & 0.87 \\
\hline $\mathrm{K}_{2} \mathrm{O}$ & 02 & 0.00 & 0.00 & 0.02 & 0.00 & 0.00 & 0.00 & 0.00 & 0.02 & 0.01 & 0.02 & 0.02 & 0.03 & 0.01 & 0.00 & 0.03 & 0.01 \\
\hline $\mathrm{Ti}$ & 0.001 & 0.000 & 0.000 & 0.008 & 0.011 & 0.006 & 0.002 & 0.025 & 0.017 & 0.017 & 0.014 & 0.007 & 0.020 & 0.009 & 0.013 & 0.030 & 0.025 \\
\hline $\mathrm{Al}_{\mathrm{IV}}$ & 0.000 & 0.000 & 0.008 & 0.051 & 0.085 & 0.032 & 0.041 & 0.098 & 0.058 & 0.055 & 0.078 & 0.048 & 0.124 & 0.061 & 0.101 & 0.129 & 0.097 \\
\hline $\mathrm{Al}_{\mathrm{VI}}$ & - & - & - & 0.000 & 0.003 & 0.025 & 0.005 & 0.007 & 0.060 & 0.055 & 0.033 & 0.017 & 0.016 & 0.000 & 0.032 & 0.108 & 0.098 \\
\hline $\mathrm{Fe}$ & 0.692 & 0.711 & 0.633 & 0.574 & 0.433 & 0.619 & 0.577 & 0.249 & 0.234 & 0.217 & 0.256 & 0.192 & 0.258 & 0.254 & 0.277 & 0.203 & 0.211 \\
\hline $\mathrm{Ni}$ & 0.001 & 0.001 & 0.002 & 0.001 & 0.003 & 0.000 & 0.001 & 0.001 & 0.001 & 0.000 & 0.000 & 0.001 & 0.002 & 0.001 & 0.000 & 0.000 & 0.003 \\
\hline $\mathrm{Mn}$ & 0.000 & 0.009 & 0.008 & 0.017 & 0.001 & 0.015 & 0.013 & 0.000 & 0.006 & 0.006 & 0.006 & 0.009 & 0.003 & 0.008 & 0.007 & 0.005 & 0.006 \\
\hline $\mathrm{Mg}$ & 1.339 & 1.274 & 1.336 & 1.370 & 1.445 & 1.317 & 1.391 & 0.910 & 0.954 & 0.851 & 0.922 & 0.837 & 0.905 & 0.950 & 1.051 & 0.812 & 0.837 \\
\hline $\mathrm{Ca}$ & 0.000 & 0.001 & 0.009 & 0.033 & 0.098 & 0.018 & 0.010 & 0.774 & 0.695 & 0.813 & 0.720 & 0.901 & 0.736 & 0.760 & 0.629 & 0.752 & 0.745 \\
\hline $\mathrm{Na}$ & 0.002 & 0.000 & 0.005 & 0.003 & 0.004 & 0.000 & 0.000 & 0.028 & 0.021 & 0.032 & 0.035 & 0.031 & 0.051 & 0.025 & 0.029 & 0.080 & 0.062 \\
\hline $\mathrm{K}$ & 0.001 & 0.000 & 0.000 & 0.001 & 0.000 & 0.000 & 0.000 & 0.000 & 0.001 & 0.001 & 0.001 & 0.001 & 0.001 & 0.000 & 0.000 & 0.001 & 0.001 \\
\hline Cations & 3.018 & 2.998 & 2.999 & 3.998 & 3.998 & 4.000 & 3.999 & 3.994 & 3.989 & 3.992 & 3.987 & 3.996 & 3.992 & 3.999 & 4.038 & 3.991 & 3.988 \\
\hline
\end{tabular}

\begin{tabular}{|c|c|c|c|c|c|c|c|c|c|c|c|c|c|c|c|c|c|c|}
\hline \multirow{3}{*}{$\begin{array}{l}\text { Sample } \\
\text { Analysis }\end{array}$} & \multicolumn{7}{|c|}{ Plagioclase } & \multicolumn{7}{|c|}{ Amphibole } & \multicolumn{4}{|c|}{ Phlogopite } \\
\hline & \multicolumn{3}{|c|}{$107038 \mathrm{~L}$} & \multicolumn{4}{|c|}{$108585 \mathrm{~L}$} & \multicolumn{4}{|c|}{$107038 \mathrm{~L}$} & \multicolumn{3}{|c|}{$108585 \mathrm{~L}$} & \multicolumn{2}{|c|}{$107038 \mathrm{~L}$} & \multicolumn{2}{|c|}{$108585 \mathrm{~L}$} \\
\hline & 160 & 161 & 173 & 1 & 5 & 187 & 188 & $7 b$ & $8 \mathrm{a}$ & 56 & 182 & 20 & 67 & 82 & $8 b$ & 34 & $10 \mathrm{e}$ & 76 \\
\hline $\mathrm{TiO}_{2}$ & 0.07 & 0.04 & 0.07 & 0.07 & 0.13 & 0.03 & 0.00 & 3.25 & 2.71 & 4.48 & 1.50 & 0.66 & 2.04 & 3.88 & 4.37 & 3.14 & 4.44 & 2.58 \\
\hline $\mathrm{Al}_{2} \mathrm{O}_{3}$ & 27.71 & 24.30 & 30.77 & 30.78 & 27.57 & 24.75 & 21.12 & 11.75 & 10.49 & 12.19 & 11.59 & 13.85 & 12.23 & 11.92 & 15.37 & 16.08 & 14.38 & 18.02 \\
\hline $\mathrm{MnO}$ & 0.02 & 0.01 & 0.03 & 0.00 & 0.06 & 0.00 & 0.03 & 0.02 & 0.07 & 0.13 & 0.11 & 0.16 & 0.15 & 0.09 & 0.00 & 0.00 & 0.18 & 0.00 \\
\hline $\mathrm{IgO}$ & 0.00 & 0.00 & 0.00 & .00 & 00 & 0.00 & 0.0 & 13.64 & 15.30 & 13.59 & 14. & 15.38 & 14. & 12. & 16.73 & 19.39 & 12.81 & 20.03 \\
\hline $\mathrm{aC}$ & 9.46 & 5.94 & 12.83 & 13.96 & 9.33 & 6.18 & 2.38 & 11.18 & 11.25 & 11.33 & 10.91 & 11.30 & 11.00 & 11.40 & 0.02 & 0.01 & 0.13 & 0.06 \\
\hline $\mathrm{Na}_{2} \mathrm{O}$ & 6.39 & 8.47 & 4.05 & 4.07 & 6.67 & 8.41 & 10.77 & 2.45 & 2.48 & 2.37 & 2.70 & 2.65 & 2.62 & 2.5 & 0.64 & 0.94 & 0.21 & 1.22 \\
\hline $\mathrm{K}_{2} \mathrm{O}$ & 0.05 & 0.03 & 0.04 & 0.02 & 0.08 & 0.08 & 0.17 & 1.01 & 0.57 & 1.21 & 0.4 & 0.29 & 0.34 & 0.9 & 8.67 & 8.31 & 9.04 & 8.19 \\
\hline Total & 100.5 & 100.3 & 100.1 & 99.8 & 99.7 & 100.5 & 100.0 & 97.0 & 96.3 & 96.1 & 96.2 & 96.7 & 96.3 & 96.1 & 94.6 & 94.0 & 94.9 & 94.2 \\
\hline $\mathrm{Al}_{\mathrm{VI}}$ & - & - & - & - & - & - & - & 0.708 & 0.679 & 0.216 & 0.709 & 0.988 & 0.486 & 0.262 & 0.155 & 0.247 & 0.044 & 0.377 \\
\hline $\mathrm{Fe}$ & 0.001 & 0.006 & 0.005 & 0.009 & 0.038 & 0.000 & 0.004 & 1.437 & 1.184 & 1.296 & 1.172 & 1.124 & 1.227 & 1.454 & 1.431 & 1.065 & 2.297 & 0.940 \\
\hline $\mathrm{Ni}$ & - & - & - & - & - & - & - & - & - & - & - & - & - & - & - & - & - & - \\
\hline $\mathrm{Mn}$ & 0.003 & 0.002 & 0.005 & 0.000 & 0.009 & 0.000 & 0.005 & 0.003 & 0.010 & 0.016 & 0.013 & 0.021 & 0.019 & 0.01 & 0.000 & 0.000 & 0.023 & 0.000 \\
\hline $\mathrm{Mg}$ & 0.000 & 0.000 & 0.000 & 0.000 & 0.000 & 0.000 & 0.012 & 3.141 & 3.503 & 3.040 & 3.344 & 3.447 & 3.250 & 2.883 & 3.679 & 4.226 & 2.918 & 4.344 \\
\hline $\mathrm{Ca}$ & 1.811 & 1.127 & 2.487 & 2.734 & 1.808 & 1.172 & 0.449 & 1.851 & 1.850 & 1.822 & 1.750 & 1.819 & 1.737 & 1.840 & 0.003 & 0.001 & 0.021 & 0.009 \\
\hline $\mathrm{Na}$ & 2.214 & 2.912 & 1.422 & 1.440 & 2.337 & 2.890 & 3.685 & 0.735 & 0.740 & 0.689 & 0.784 & 0.772 & 0.749 & 0.742 & 0.183 & 0.266 & 0.063 & 0.345 \\
\hline K & 0.012 & 0.007 & 0.009 & 0.005 & 0.018 & 0.017 & 0.039 & 0.199 & 0.112 & 0.232 & 0.088 & 0.055 & 0.063 & 0.191 & 1.632 & 1.550 & 1.761 & 1.521 \\
\hline Cations & 20.03 & 20.02 & 19.95 & 20.10 & 20.16 & 20.05 & 20.12 & 16.45 & 16.39 & 15.82 & 16.03 & 16.30 & 15.76 & 15.82 & 15.57 & 15.70 & 15.64 & 15.82 \\
\hline
\end{tabular}

Table 2.- Major element composition (wt.\%) of representative minerals from SCS gabbros 


\begin{tabular}{|c|c|c|c|c|c|c|c|c|c|c|c|c|c|c|c|c|c|}
\hline \multirow{4}{*}{$\begin{array}{l}\text { Sample } \\
\text { Analysis }\end{array}$} & \multicolumn{3}{|c|}{ olivine } & \multicolumn{4}{|c|}{ orthopyroxene } & \multicolumn{10}{|c|}{ clinopyroxene } \\
\hline & \multirow{3}{*}{$\begin{array}{c}\text { Talavera } \\
107038 \mathrm{~L} \\
16 \mathrm{c} \\
\end{array}$} & \multirow{2}{*}{\multicolumn{2}{|c|}{$\begin{array}{c}\text { La Solanilla } \\
108585 \mathrm{~L} \\
\end{array}$}} & \multirow{2}{*}{\multicolumn{2}{|c|}{$\begin{array}{c}\text { Talavera } \\
107038 \mathrm{~L}\end{array}$}} & \multirow{2}{*}{\multicolumn{2}{|c|}{$\begin{array}{c}\text { La Solanilla } \\
108585 \mathrm{~L} \\
\end{array}$}} & \multirow{2}{*}{\multicolumn{5}{|c|}{$\begin{array}{c}\text { Talavera } \\
107038 \mathrm{~L}\end{array}$}} & \multicolumn{5}{|c|}{ La Solanilla } \\
\hline & & & & & & & & & & & & & & & $08585 \mathrm{~L}$ & & \\
\hline & & $08 \mathrm{~d}$ & $10 \mathrm{c}$ & $04 \mathrm{a}$ & $07 \mathrm{c}$ & $05 \mathrm{~d}$ & $07 d$ & $015 \mathrm{a}$ & 166 & 171 & 247 & 250 & 11 & 13 & 65 & 136 & 138 \\
\hline $\mathrm{Ba}$ & 0.316 & 0.068 & 0.054 & 0.134 & 0.079 & 0.065 & 1.25 & 14.8 & 0.110 & 9.69 & 5.31 & 6.63 & 6.93 & 16.7 & 1.19 & 5.14 & 1.54 \\
\hline $\mathrm{Rb}$ & 0.068 & 0.102 & 0.058 & 0.061 & 0.056 & 0.055 & 0.371 & 1.54 & 0.040 & 1.49 & 1.00 & 6.81 & 0.150 & 0.300 & 0.037 & 0.610 & 0.390 \\
\hline $\mathrm{Sr}$ & 0.197 & 0.130 & 0.421 & 0.454 & 0.329 & 0.229 & 0.118 & 27.4 & 14.7 & 20.9 & 18.4 & 19.4 & 20.3 & 62.5 & 15.1 & 29.8 & 25.2 \\
\hline $\mathrm{Pb}$ & 0.080 & 0.046 & 0.051 & 0.069 & 0.056 & 0.037 & 0.052 & 0.658 & 0.400 & 0.680 & 0.870 & 1.860 & 0.410 & 0.770 & 0.046 & 0.340 & 0.410 \\
\hline Th & 0.019 & 0.055 & 0.025 & 0.022 & 0.304 & 0.397 & 0.730 & 0.842 & 0.310 & 1.06 & 1.01 & 0.810 & 0.610 & 0.730 & 0.102 & 0.160 & 0.440 \\
\hline $\mathrm{U}$ & 0.014 & 0.019 & 0.010 & 0.015 & 0.037 & 0.059 & 0.166 & 0.102 & 0.090 & 0.170 & 0.140 & 0.130 & 0.050 & 0.050 & 0.013 & 0.020 & 0.040 \\
\hline $\mathrm{Zr}$ & 0.716 & 0.705 & 0.531 & 10.1 & 8.65 & 30.0 & 11.2 & 81.3 & 24.6 & 59.4 & 72.9 & 99.8 & 38.4 & 64.5 & 21.6 & 48.7 & 56.9 \\
\hline $\mathrm{Nb}$ & 0.044 & 0.022 & 0.015 & 0.036 & 0.022 & 0.156 & 0.100 & 0.447 & 0.050 & 0.530 & 0.810 & 0.170 & 0.060 & 0.320 & 0.030 & 0.200 & 0.050 \\
\hline Y & 0.094 & 0.264 & 0.269 & 13.2 & 8.03 & 11.1 & 3.56 & 63.0 & 24.0 & 37.7 & 38.6 & 42.6 & 34.5 & 37.5 & 23.8 & 35.9 & 42.0 \\
\hline $\mathrm{Sc}$ & 6.63 & 3.08 & 4.77 & 29.1 & 75.6 & 57.5 & 6.06 & 181 & 103 & 115 & 94.1 & 93.6 & 122 & 127 & 120 & 145 & 141 \\
\hline Co & 221 & 207 & 184 & 71.8 & 79.1 & 76.9 & 70.8 & 30.7 & 43.4 & 44.1 & 37.4 & 41.8 & 46.1 & 50.8 & 34.7 & 41.2 & 41.5 \\
\hline V & 2.68 & 3.00 & 3.79 & 65.6 & 265 & 91.6 & 52.7 & 540 & 607 & 657 & 496 & 276 & 568 & 545 & 333 & 637 & 656 \\
\hline $\mathrm{Cr}$ & 3.48 & 189 & 134 & 68.5 & 766 & 5.95 & 4.07 & 751 & 5710 & 6090 & 4500 & 1050 & 2100 & 533 & 2130 & 2860 & 3180 \\
\hline $\mathrm{Ta}$ & 0.003 & 0.013 & 0.018 & 0.019 & 0.007 & 0.017 & 0.015 & 0.042 & 0.010 & 0.030 & 0.060 & 0.010 & 0.010 & 0.010 & 0.005 & 0.020 & 0.010 \\
\hline $\mathrm{Hf}$ & 0.089 & 0.107 & 0.092 & 0.409 & 0.322 & 1.27 & 0.824 & 3.44 & 1.11 & 1.54 & 2.00 & 3.70 & 1.34 & 2.20 & 0.823 & 2.12 & 2.17 \\
\hline $\mathrm{La}$ & 0.016 & 0.049 & 0.035 & 0.150 & 0.193 & 0.468 & 0.132 & 6.92 & 1.91 & 7.14 & 6.85 & 6.94 & 2.90 & 3.94 & 1.06 & 1.70 & 2.38 \\
\hline $\mathrm{Ce}$ & 0.018 & 0.128 & 0.046 & 0.354 & 0.498 & 0.918 & 0.254 & 16.7 & 7.40 & 26.6 & 24.1 & 26.2 & 8.99 & 12.3 & 3.24 & 6.32 & 8.35 \\
\hline $\operatorname{Pr}$ & 0.012 & 0.029 & 0.011 & 0.094 & 0.068 & 0.179 & 0.040 & 3.53 & 1.36 & 4.15 & 4.19 & 4.64 & 1.61 & 2.07 & 0.714 & 1.38 & 1.56 \\
\hline $\mathrm{Nd}$ & 0.176 & 0.153 & 0.242 & 0.849 & 0.568 & 1.08 & 0.405 & 22.5 & 8.45 & 21.4 & 21.5 & 22.9 & 8.55 & 12.7 & 4.74 & 8.32 & 9.64 \\
\hline $\mathrm{Sm}$ & 0.063 & 0.073 & 0.013 & 0.437 & 0.321 & 0.692 & 0.145 & 8.10 & 2.98 & 5.58 & 5.94 & 6.66 & 3.15 & 4.22 & 2.03 & 3.46 & 3.93 \\
\hline $\mathrm{Eu}$ & 0.021 & 0.020 & 0.026 & 0.052 & 0.052 & 0.073 & 0.027 & 1.46 & 0.740 & 1.120 & 1.100 & 0.920 & 0.720 & 0.830 & 0.600 & 1.01 & 1.21 \\
\hline Gd & 0.164 & 0.077 & 0.062 & 0.812 & 0.516 & 0.912 & 0.259 & 10.6 & 3.84 & 6.01 & 6.66 & 7.48 & 4.89 & 5.44 & 3.37 & 5.12 & 5.75 \\
\hline $\mathrm{Tb}$ & 0.012 & 0.015 & 0.011 & 0.222 & 0.127 & 0.202 & 0.051 & 1.71 & 0.670 & 1.00 & 1.17 & 1.24 & 0.900 & 0.960 & 0.571 & 0.960 & 1.05 \\
\hline Dy & 0.030 & 0.048 & 0.048 & 1.69 & 1.10 & 1.91 & 0.489 & 12.4 & 4.77 & 6.89 & 7.70 & 8.09 & 6.51 & 7.30 & 4.42 & 6.76 & 7.56 \\
\hline Но & 0.010 & 0.014 & 0.014 & 0.410 & 0.295 & 0.419 & 0.144 & 2.53 & 0.920 & 1.48 & 1.56 & 1.61 & 1.33 & 1.49 & 0.930 & 1.44 & 1.64 \\
\hline $\mathrm{Er}$ & 0.061 & 0.039 & 0.055 & 1.69 & 1.19 & 1.54 & 0.462 & 6.55 & 2.73 & 4.07 & 4.46 & 4.53 & 4.08 & 4.12 & 2.76 & 4.24 & 4.89 \\
\hline $\mathrm{Tm}$ & 0.014 & 0.022 & 0.014 & 0.373 & 0.231 & 0.293 & 0.076 & 0.955 & 0.370 & 0.600 & 0.570 & 0.650 & 0.600 & 0.590 & 0.414 & 0.570 & 0.690 \\
\hline $\mathrm{Yb}$ & 0.109 & 0.055 & 0.095 & 3.10 & 1.75 & 2.83 & 0.726 & 5.67 & 2.32 & 4.24 & 3.79 & 4.54 & 3.98 & 4.44 & 2.78 & 4.21 & 4.55 \\
\hline $\mathrm{Lu}$ & 0.044 & 0.019 & 0.013 & 0.575 & 0.260 & 0.548 & 0.127 & 0.838 & 0.330 & 0.580 & 0.560 & 0.630 & 0.580 & 0.550 & 0.409 & 0.590 & 0.650 \\
\hline
\end{tabular}

Plagioclase

Amphibole

Phlogopite

\begin{tabular}{|c|c|c|c|c|c|c|c|c|c|c|c|c|c|c|c|c|c|c|}
\hline \multirow{3}{*}{$\begin{array}{l}\text { Sample } \\
\text { Analysis }\end{array}$} & & & \\
\hline & \multicolumn{3}{|c|}{$107038 \mathrm{~L}$} & \multicolumn{4}{|c|}{$108585 \mathrm{~L}$} & \multicolumn{4}{|c|}{$107038 \mathrm{~L}$} & \multicolumn{3}{|c|}{$108585 \mathrm{~L}$} & \multicolumn{2}{|c|}{$107038 \mathrm{~L}$} & \multicolumn{2}{|c|}{$108585 \mathrm{~L}$} \\
\hline & 160 & 161 & 173 & 1 & 5 & 187 & 188 & $7 \mathrm{~b}$ & $8 \mathrm{a}$ & 56 & 182 & 20 & 67 & 82 & $8 \mathrm{~b}$ & 34 & $10 \mathrm{e}$ & 76 \\
\hline $\mathrm{Ba}$ & 82.6 & 93.6 & 88.3 & 65.7 & 136 & 280 & 209 & 239 & 17.4 & 33.7 & 171 & 54.4 & 91.6 & 295 & 3140 & 1250 & 976 & 3340 \\
\hline $\mathrm{Rb}$ & 0.130 & 0.240 & 0.058 & 0.780 & 2.68 & 1.42 & 0.16 & 5.13 & 1.53 & 2.87 & 4.42 & 0.97 & 1.7 & 2.89 & 374 & 326 & 346 & 288 \\
\hline $\mathrm{Sr}$ & 538 & 447 & 606 & 706 & 729 & 822 & 369 & 92.6 & 97.6 & 51.3 & 89.9 & 48.0 & 50.6 & 95.8 & 9.07 & 19.8 & 13.9 & 31.4 \\
\hline $\mathrm{Pb}$ & 12.1 & 11.3 & 6.59 & 2.46 & 6.29 & 7.63 & 3.72 & 2.33 & 4.3 & 1.58 & 2.82 & 1.25 & 1.02 & 1.91 & 4.91 & 5.48 & 2.41 & 4.36 \\
\hline $\mathrm{Th}$ & 0.015 & 0.017 & 0.010 & 0.030 & 0.014 & 0.050 & 0.016 & 2.06 & 3.98 & 2.22 & 2.03 & 8.59 & 0.92 & 0.92 & 0.02 & 0.01 & 0.01 & 0.02 \\
\hline $\mathrm{U}$ & 0.012 & 0.011 & 0.007 & 0.010 & 0.005 & 0.050 & 0.010 & 0.58 & 0.24 & 0.29 & 0.36 & 1.25 & 0.086 & 0.055 & 0.01 & 0.01 & 0.00 & 0.01 \\
\hline $\mathrm{Zr}$ & 0.038 & 0.076 & 0.040 & 1.14 & 0.170 & 0.220 & 0.038 & 819 & 521 & 1240 & 230 & 250 & 430 & 603 & 25.3 & 12.4 & 3.84 & 2.11 \\
\hline $\mathrm{Nb}$ & 0.041 & 0.054 & 0.014 & 0.033 & 0.021 & 0.140 & 0.012 & 37.7 & 21.5 & 45.4 & 24.6 & 13.4 & 36.9 & 27.4 & 36.7 & 34.6 & 13.1 & 15.0 \\
\hline $\mathrm{Y}$ & 0.390 & 0.080 & 0.560 & 0.470 & 0.320 & 0.540 & 0.090 & 206 & 209 & 183 & 123 & 223 & 300 & 400 & 0.05 & 0.02 & 0.28 & 0.05 \\
\hline $\mathrm{Sc}$ & 2.74 & 3.00 & 2.77 & 4.11 & 4.06 & 3.73 & 3.11 & 123 & 152 & 93.0 & 95.8 & 14.3 & 59.0 & 212 & 15.8 & 11.3 & 13.1 & 8.49 \\
\hline $\mathrm{Co}$ & 0.051 & 0.085 & 0.080 & 0.310 & 0.640 & 0.140 & 0.074 & 43.9 & 48.7 & 46.5 & 57.1 & 46.9 & 43.8 & 48.4 & 92.2 & 93.0 & 93.2 & 67.2 \\
\hline V & 0.330 & 0.280 & 2.44 & 2.47 & 3.87 & 0.200 & 0.090 & 438 & 922 & 334 & 609 & 95.8 & 440 & 514 & 435 & 435 & 710 & 715 \\
\hline $\mathrm{Cr}$ & 1.83 & 1.71 & 1.03 & 1.49 & 1.45 & 1.27 & 1.55 & 688 & 1600 & 486 & 565 & 2.9 & 4.66 & 17.6 & 1370 & 354 & 43.0 & 128 \\
\hline Ta & 0.016 & 0.019 & 0.001 & 0.012 & 0.012 & 0.040 & 0.018 & 2.67 & 1.43 & 3.91 & 1.36 & 0.8 & 3.3 & 1.81 & 2.45 & 2.70 & 1.20 & 1.27 \\
\hline Hf & 0.033 & 0.093 & 0.027 & 0.059 & 0.094 & 0.076 & 0.062 & 24.8 & 22.5 & 42.0 & 7.86 & 9.61 & 23.8 & 22.0 & 1.18 & 0.563 & 0.177 & 0.050 \\
\hline $\mathrm{La}$ & 16.9 & 9.07 & 2.96 & 1.71 & 1.56 & 6.95 & 7.71 & 44.3 & 28.8 & 47.8 & 26.5 & 75.7 & 37.9 & 47.3 & 0.015 & 0.013 & 0.256 & 0.029 \\
\hline $\mathrm{Ce}$ & 20.6 & 11.1 & 5.29 & 3.13 & 2.80 & 8.43 & 7.86 & 120 & 80.2 & 140 & 93.3 & 216 & 122 & 138 & 0.011 & 0.010 & 0.401 & 0.033 \\
\hline $\operatorname{Pr}$ & 1.52 & 0.710 & 0.570 & 0.310 & 0.290 & 0.650 & 0.510 & 23.0 & 13.3 & 26.1 & 15.8 & 30.6 & 24.4 & 27.7 & 0.007 & 0.006 & 0.040 & 0.009 \\
\hline $\mathrm{Nd}$ & 4.45 & 2.76 & 1.99 & 1.01 & 1.29 & 2.06 & 1.41 & 126 & 75.8 & 128 & 82.2 & 144 & 129 & 157 & 0.079 & 0.082 & 0.231 & 0.136 \\
\hline $\mathrm{Sm}$ & 0.510 & 0.097 & 0.340 & 0.110 & 0.110 & 0.220 & 0.140 & 34.7 & 26.9 & 34.1 & 24.1 & 35.3 & 40.3 & 50.6 & 0.053 & 0.079 & 0.061 & 0.070 \\
\hline $\mathrm{Eu}$ & 1.67 & 1.34 & 0.970 & 0.670 & 1.27 & 3.10 & 2.03 & 4.23 & 3.71 & 3.62 & 3.82 & 5.46 & 4.04 & 10.1 & 0.034 & 0.062 & 0.079 & 0.193 \\
\hline $\mathrm{Gd}$ & 0.180 & 0.190 & 0.250 & 0.076 & 0.110 & 0.130 & 0.090 & 37.7 & 32.5 & 35.0 & 25.9 & 38.1 & 44.2 & 63.2 & 0.048 & 0.113 & 0.050 & 0.075 \\
\hline $\mathrm{Tb}$ & 0.013 & 0.020 & 0.030 & 0.019 & 0.010 & 0.013 & 0.016 & 5.74 & 5.70 & 5.41 & 3.91 & 5.99 & 7.69 & 11.0 & 0.011 & 0.008 & 0.009 & 0.010 \\
\hline Dy & 0.100 & 0.053 & 0.100 & 0.130 & 0.090 & 0.080 & 0.040 & 39.1 & 39.3 & 35.5 & 25.3 & 40.4 & 54.6 & 76.6 & 0.070 & 0.031 & 0.043 & 0.051 \\
\hline Ho & 0.015 & 0.018 & 0.020 & 0.020 & 0.010 & 0.020 & 0.010 & 7.99 & 8.06 & 7.09 & 4.96 & 8.37 & 11.5 & 16.0 & 0.005 & 0.006 & 0.012 & 0.006 \\
\hline $\mathrm{Er}$ & 0.040 & 0.048 & 0.100 & 0.090 & 0.030 & 0.061 & 0.061 & 21.9 & 23.6 & 18.7 & 13.3 & 23.9 & 34.4 & 45.8 & 0.047 & 0.049 & 0.028 & 0.028 \\
\hline $\mathrm{Tm}$ & 0.007 & 0.013 & 0.008 & 0.010 & 0.016 & 0.018 & 0.006 & 2.96 & 3.23 & 2.60 & 1.77 & 3.40 & 5.18 & 6.71 & 0.011 & 0.008 & 0.005 & 0.008 \\
\hline $\mathrm{Yb}$ & 0.058 & 0.078 & 0.105 & 0.079 & 0.060 & 0.080 & 0.076 & 19.5 & 21.0 & 16.5 & 11.1 & 22.0 & 33.0 & 42.6 & 0.038 & 0.056 & 0.052 & 0.058 \\
\hline $\mathrm{Lu}$ & 0.013 & 0.015 & 0.011 & 0.010 & 0.016 & 0.020 & 0.008 & 2.77 & 2.95 & 2.19 & 1.49 & 3.01 & 4.66 & 6.07 & 0.011 & 0.006 & 0.009 & 0.006 \\
\hline
\end{tabular}

Table 3.- Trace element composition (ppm) of representative minerals from SCS gabbros 
to ophitic texture. Interstitial minerals are clinopyroxene, amphibole, phlogopite and ilmenite, together with Na-rich plagioclase overgrowths (Fig. 2a, b). Amphibole and phlogopite are relatively abundant $(\sim 9 \%)$ and exhibit a relatively large size (Fig. 2c, d) (similar to that of clinopyroxene; i.e. 1-3 $\mathrm{mm})$. A pale green amphibole is also present in much lower proportions, generally forming thin margins around large brown amphiboles (Fig. 2d).

Early magmatic minerals from La Solanilla sample (olivine and plagioclase) constitute more than $70 \%$ of the rock. Oli- vine from this gabbro is characterised by abundant $\mathrm{Cr}$-spinel inclusions and development of complex coronas formed by an inner shell of orthopyroxene (sometimes consisting of radial columnar grains) and a second shell of Ti-poor pargasitic amphibole (Fig. 2e). Orthopyroxene only appears in these thin coronas. The abundance of amphibole and phlogopite $(\sim 6 \%)$ is lower than in the Talavera sample, as well as their size $(<$ $1 \mathrm{~mm})$. While pale green amphibole is restricted to coronas around olivine, brown amphibole appears within interstitial pockets throughout the sample, associated with phlogopite
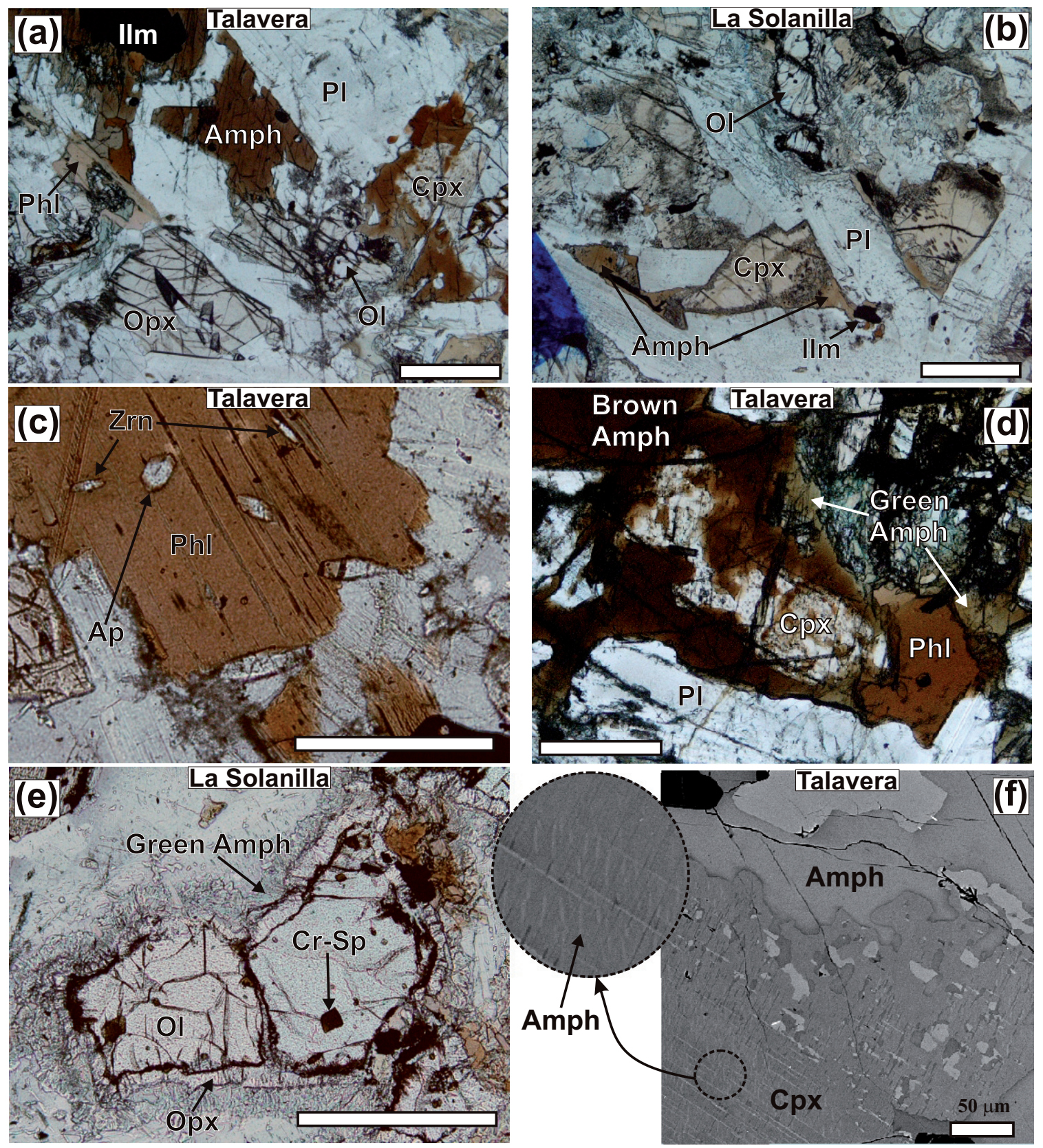

Fig. 2.- Plane polarized light (a-e) and back scattered electron (f) images showing the main textures of Talavera and La Solanilla gabbroic intrusions. a-b: General texture of Talavera and La Solanilla gabbros, respectively. c: Interstitial phlogopite with zircon and apatite inclusions (Talavera sample). d: Clinopyroxene being replaced by amphibole in Talavera sample. Note that two types of amphibole are present: a large brown amphibole and a smaller pale green amphibole (center and right respectively). e: Composite orthopyroxene-pale-green amphibole corona in olivine-plagioclase contact (La Solanilla sample). f: Back scattered electron image showing the disequilibrium texture between clinopyroxene and amphibole (Talavera sample). A detail of the reaction zone is expanded (circle), where small amphibole inclusions growing within the clinopyroxene crystal are apparent. White bar in images a-e represent $250 \mu \mathrm{m}$. 
and ilmenite (Fig. 2b). Subsolidus alteration is manifested by the formation of a secondary paragenesis mainly consisting of chlorite, sericite and a secondary green- to colourless amphibole (Mg-hornblende to actinolite and cummingtonite) at the expense of the primary minerals.

A main characteristic of both samples (although it is more notorious in the Talavera gabbro) is the common presence of reaction textures around clinopyroxene, which is variably replaced by amphibole. This is also manifested by the growth of small oriented amphibole inclusions $(2 \times 10 \mu \mathrm{m})$ within clinopyroxene rims (Fig. 2f).

The main accessory minerals of these gabbros appear within the interstitial matrix (Fig. 2c), and are represented by ilmenite (which is always included in amphibole and phlogopite), apatite, zircon, baddeleyite and titanite. Zircon crystals are more abundant in the Talavera gabbro when compared with La Solanilla (see Villaseca et al., 2011 for further details). This mineral can be found either as isolated large grains or as second-generation lamellar crystals at the ilmenite rims.

\section{Mineral composition}

\subsection{Major elements}

Major element composition of the main minerals is heterogeneous in both gabbroic intrusions (Table 2, Supp. eTable1 and Fig. 3). The mafic phases are good examples of this heterogeneity: the forsterite content of olivine ranges from $\mathrm{Fo}_{78}$ to $\mathrm{Fo}_{63}$ (see Orejana et al., 2009), and the $\mathrm{Mg} \#$ values of orthopyroxene and clinopyroxene are $0.82-0.62$ and $0.84-0.69$, respectively (Fig. 3a,b; Table 2). Orthopyroxene composition displays a marked contrast when comparing the two gabbroic intrusions, with Talavera samples yielding higher $\mathrm{Mg} \#$ (0.82-0.7) and $\mathrm{CaO}(0.85-2.5$ wt.\%) than coronitic orthopyroxene in the La Solanilla samples (Fig. 3a). Clinopyroxene exhibits the following major element values: $\mathrm{CaO}(15-22.7$ wt.\%), $\mathrm{Al}_{2} \mathrm{O}_{3}\left(1.3-7.4\right.$ wt.\%), $\mathrm{Na}_{2} \mathrm{O}\left(0.3-1.78\right.$ wt.\%) and $\mathrm{TiO}_{2}$ (0.1-1.5 wt.\%), with positive correlation between $\mathrm{Al}$, Ti and $\mathrm{Na}$ (Fig. 3b, c, d). Slight chemical differences can also be observed between clinopyroxene from the Talavera and the $\mathrm{La}$ Solanilla gabbros, with the former showing the highest $\mathrm{CaO}$, and lowest $\mathrm{Na}_{2} \mathrm{O}$ and $\mathrm{Al}_{2} \mathrm{O}_{3}$ contents (Fig. $3 \mathrm{~b}, \mathrm{c}, \mathrm{d}$ ). Clinopyroxene analyses showing the highest $\mathrm{Al}_{2} \mathrm{O}_{3}, \mathrm{TiO}_{2}$ and $\mathrm{Na}_{2} \mathrm{O}$ values in the La Solanilla gabbro (Fig. 3c, d) represent areas partially transformed to amphibole.

Plagioclase is characterised by a strong chemical variation, with anorthite values from $\mathrm{An}_{82}$ to $\mathrm{An}_{10}, \mathrm{SiO}_{2}=42-66$ wt.\% and $\mathrm{Al}_{2} \mathrm{O}_{3}=21.1-34$ wt.\% (Fig. 3e, f). Amphibole has a heterogeneous composition in $\mathrm{Mg} \#(0.62-0.78), \mathrm{TiO}_{2}$ (0-4.7 wt.\%), $\mathrm{Al}_{2} \mathrm{O}_{3}$ (10.4-16 wt.\%), $\mathrm{Na}_{2} \mathrm{O}$ (1.8-3.05 wt.\%) and $\mathrm{K}_{2} \mathrm{O}(0.2-1.15$ wt.\%) (Fig. 3g, h, i), and displays positive correlations for $\mathrm{Ti}-\mathrm{K}$ and $\mathrm{Na}-\mathrm{Al}$, and negative correlation for Ti-Mg\# (Fig. 3g, h, i). It can be classified as pargasite, magnesiohastingsite, magnesiohornblende and kaersutite (following Leake et al., 1997 criteria). Phlogopite also shows a significant within-sample variation in $\mathrm{Mg} \#(0.56-0.86)$, $\mathrm{TiO}_{2}$ (0.32-4.7 wt.\%), $\mathrm{Al}_{2} \mathrm{O}_{3}\left(14.4-18\right.$ wt.\%), $\mathrm{Na}_{2} \mathrm{O}(0.2-1.3$ wt.\%) and $\mathrm{K}_{2} \mathrm{O}$ (7.4-9.1 wt.\%) (Fig. 3j, k, 1). It also exhibits negative correlation between $\mathrm{Ti}$ and $\mathrm{Mg} \#$ and $\mathrm{Al}$ (Fig. 3j, k), so that a few phlogopite crystals display very low $\mathrm{TiO}_{2}$ values (down to 0.32 wt.\%) with $\mathrm{Mg \#} \mathrm{higher} \mathrm{than} 0.78$ (Fig. 3j).

Some of the main minerals present chemical zoning. In clinopyroxene crystals, the $\mathrm{TiO}_{2}, \mathrm{Al}_{2} \mathrm{O}_{3}$ and $\mathrm{Na}_{2} \mathrm{O}$ concentration decreases from core to rim (Fig. 4). The chemical heterogeneity of plagioclase is also manifested in marked core to rim zoning patterns within a single crystal (e.g., anorthite from $\mathrm{An}_{61}$ to $\mathrm{An}_{10}$ ). The most An-depleted plagioclase corresponds to rims and anhedral interstitial crystals adjacent to subhedral plagioclase prisms (Fig. 4). Analysed amphibole crystals do not display important zoning patterns, but they reveal that two broad compositional poles can be distinguished regarding their major element contents (with a few intermediate analyses): 1) large brown amphibole with high $\mathrm{TiO}_{2}$ and $\mathrm{K}_{2} \mathrm{O}$, and 2) brown to green amphibole with lower concentrations of these elements (Fig. 5). Mg\# can be relatively high in the low-Ti-K amphiboles (Fig. 3i).

\subsection{Trace elements}

The complete set of trace element data is available in Supp. eTable 2. Olivine trace element contents are characterised by moderate to low values for the transition metals: $\mathrm{Ni}$ (212-1570 ppm), Cr (up to 190 ppm), Co (184-242 ppm), V (0.6-3.8 ppm) and Sc (3-7.7 ppm) (Table 2 and 3). Olivine from the Talavera sample displays higher $\mathrm{Sc}$ and Ni values, and lower $\mathrm{Cr}$ and $\mathrm{V}$, with respect to those from La Solanilla gabbro (Tables 1 and 2).

The large locally poikilitic orthopyroxene crystals of Talavera sample (107038L) yield a variable compositional range with high $\mathrm{Cr}$ (68-4410 ppm) contents, which correlate positively with $\mathrm{Mg} \#$ (Fig. 6a). Coronitic orthopyroxene in the La Solanilla sample $(108585 \mathrm{~L})$ is characterised by very low Cr (4-14 ppm), but higher concentrations of many incompatible elements: $\mathrm{Ba}$ (up to $1.5 \mathrm{ppm}$ ), $\mathrm{Rb}$ (up to $0.4 \mathrm{ppm}$ ), Th (0.4-0.73 ppm), Zr (11-30 ppm), Nb (up to $0.16 \mathrm{ppm}$ ) and LREE (e.g. Ce up to $0.92 \mathrm{ppm}$ ), when compared with Talavera orthopyroxene (Fig. 6a, b, c). Primitive mantle-normalised plots of trace element abundances of orthopyroxene display a consistent fractionation in the REE towards lower LREE and characteristic positive $\mathrm{Th}-\mathrm{U}$ peaks and $\mathrm{Sr}-\mathrm{Eu}$ negative anomalies (Fig. 6d).

The main differences in plagioclase trace element contents between the two samples are the higher $\mathrm{Rb}$ and $\mathrm{Sr}$, and lower LREE in La Solanilla with respect to Talavera gabbro (Table 3; Fig. 6e, f, g). In both cases there is a general trend of decreasing $\mathrm{Sr}$ and increasing $\mathrm{Ba}$ and LREE associated with higher $\mathrm{Na}_{2} \mathrm{O}$ contents (Fig. 6f, g). This variation is mostly 

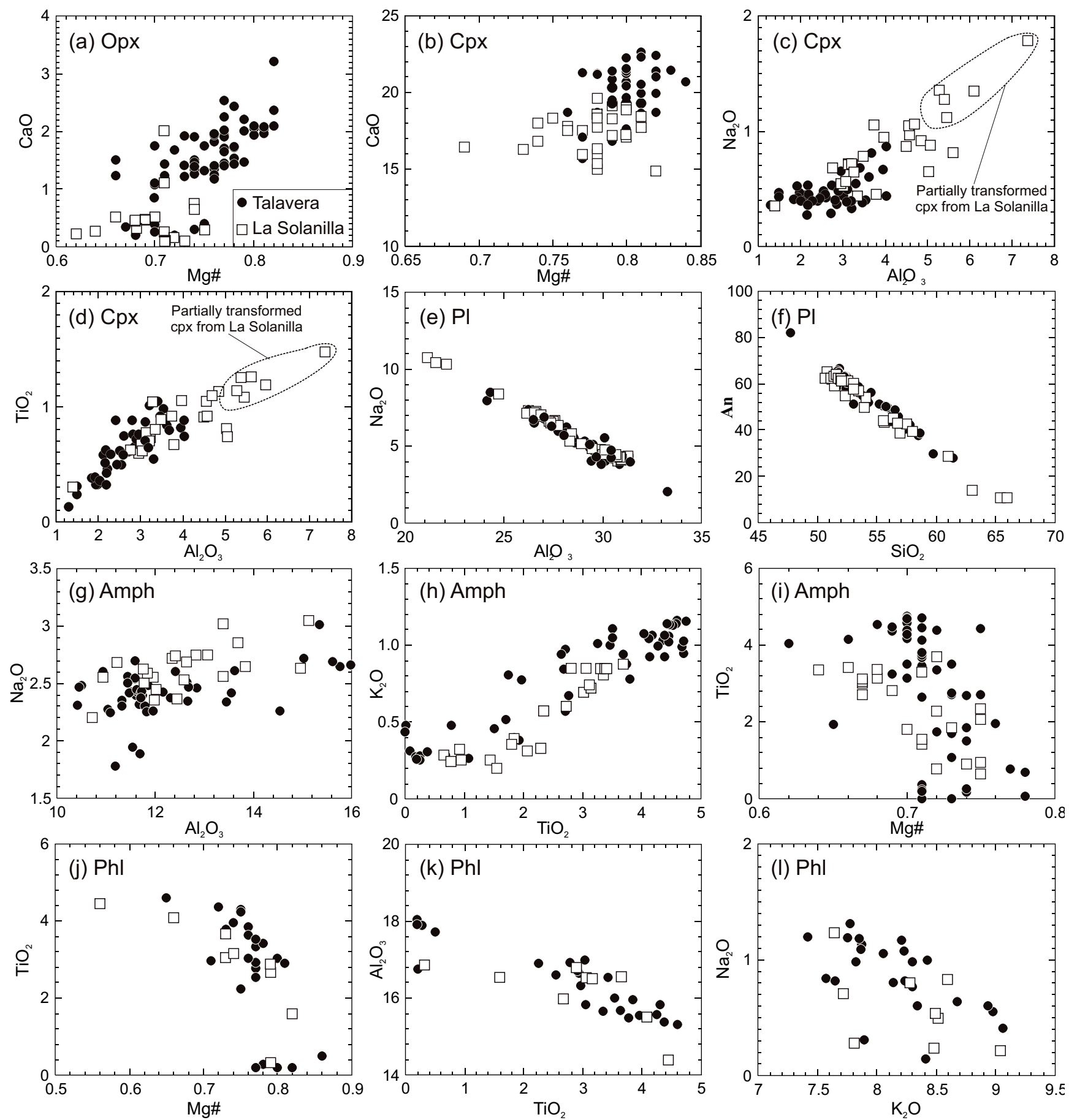

Fig. 3.- Major element composition (wt.\%) of (a) orthopyroxene, (b-d) clinopyroxene, (e-f) plagioclase, (g-i) amphibole and (j-1) phlogopite from the Talavera and La Solanilla gabbros. Clinopyroxene analyses corresponding to mineral areas partially transformed to amphibole have been indicated in diagrams c and d. Part of the orthopyroxene data plotted in diagram a have been extracted from Orejana et al. (2009).

mimicked by core to rim trace element zoning within a single euhedral crystal, but a significant drop in Ba and LREE is characteristic of extremely Na-rich plagioclase overgrowths (Fig. 4). Plagioclase also shows fractionated LREE chondrite-normalised patterns (La up to 80x chondrite abundances; Fig. 6g).

Clinopyroxene from Talavera shows higher $\mathrm{Cr}$, Th and $\mathrm{U}$ contents (e.g., Cr up to $6170 \mathrm{ppm}$, Th up to $2.86 \mathrm{ppm}$ and $\mathrm{U}$ up to 0.3 ppm; Fig. 7a, b) with respect to La Solanilla (Table 3). LREE and HFSE (high field strength elements) do not differ significantly between both intrusions (Fig. 7c, d), but HREE (heavy REE) are higher in cpx of the La Solanilla gabbro (Fig. 7e). Most incompatible trace elements in La Solanilla clinopyroxene ( $\mathrm{Ba}, \mathrm{Th}, \mathrm{Pb}, \mathrm{Zr}, \mathrm{Hf}, \mathrm{Y}, \mathrm{REE})$ are negatively correlated when using $\mathrm{Cr}$ content as a differentiation index (see examples in Fig. 7a, b, c, d). This general trend is similar 

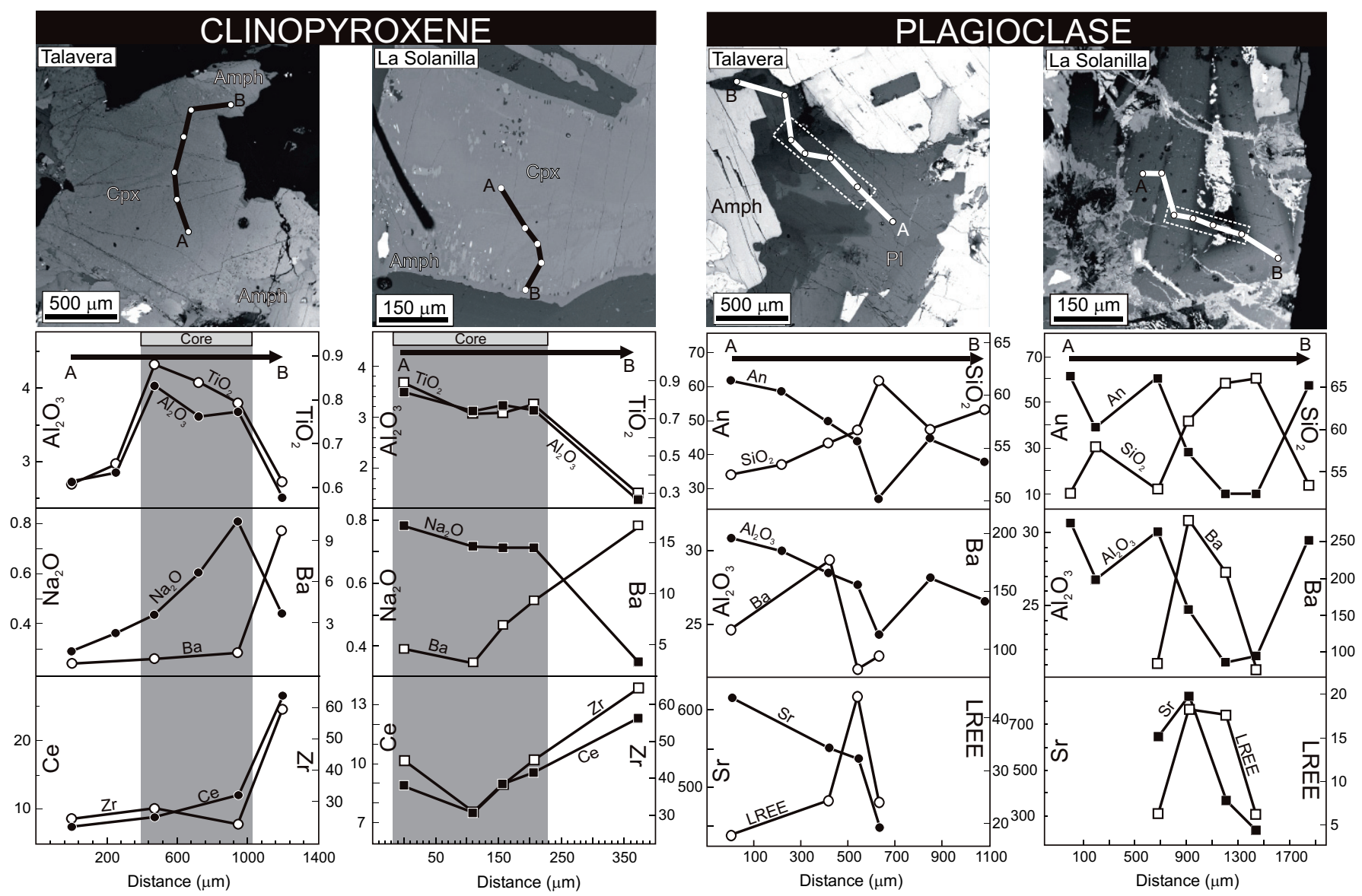

Fig. 4.- Major (wt.\%) and trace (ppm) element zoning profiles within single clinopyroxene (left) and plagioclase (right) crystals from Talavera and La Solanilla samples. Back scattered electron images at the top of each column illustrate the location of the analysis spots (white circles). Clinopyroxene core analyses in the plots are shown with a grey band. Trace element concentrations in plagioclase have been determined only in the spots located within the white dotted rectangle. Note that analyses from La Solanilla sample have been performed in several plagioclase prisms, thus causing the observed irregular compositional patterns.

to that depicted by core to rim profiles, and it is interesting to remark that analyses with the highest incompatible trace elements values correspond with partially altered clinopyroxene (areas replaced by amphibole). On the contrary, clinopyroxene from Talavera is characterised by: 1) a fairly variable trace element concentration in analyses displaying very high $\mathrm{Cr}$ contents, 2) a slight tendency of $\mathrm{Ba}, \mathrm{Th}, \mathrm{U}, \mathrm{Pb}, \mathrm{Zr}, \mathrm{Nb}, \mathrm{V}$ and LREE contents to decrease towards lower $\mathrm{Cr}$, and 3 ) the presence of a reduced group of spots with low $\mathrm{Cr}$ values but relative high incompatible trace element concentrations $(\mathrm{Ba}$, $\mathrm{U}, \mathrm{Th}, \mathrm{Pb}, \mathrm{Zr}$, Hf, Y, REE), as described in the La Solanilla gabbros (Fig. 7a, b, c, d). These latter analyses represent partially transformed zones in contact with interstitial amphiboles.

Clinopyroxene chondrite-normalised REE patterns are almost flat, but exhibit a wide compositional range and a variable degree of LREE fractionation (Fig. 7e). They always show Eu negative anomaly. The Primitive Mantle-normalised patterns display characteristic $\mathrm{Nb}-\mathrm{Ta}$, $\mathrm{Sr}$ and Ti negative anomalies (Fig. 7f). Zoning patterns in both samples are in agreement with development of rims in reaction fronts (and also inner areas) displaying lower $\mathrm{Cr}$ and higher values for incompatible elements (Fig. 4). It must be noted that, exclud- ing partially transformed clinopyroxene areas, the extreme heterogeneity of incompatible elements in Talavera sample, such as $\mathrm{Th}, \mathrm{U}, \mathrm{Pb}, \mathrm{Zr}$, Nb or LREE, corresponds with analyses displaying the highest $\mathrm{Cr}$ concentrations $(>5000 \mathrm{ppm})$ (Fig. 7a, b, c, d).

$\mathrm{Cr}$ content in amphibole from Talavera (249-796 ppm) is again significantly higher than in La Solanilla gabbro $(<96$ ppm) (Table 3). The trace element composition of amphibole from both intrusions shows a broad tendency of the low Ti-K group to show lower $\mathrm{Ba}, \mathrm{Rb}, \mathrm{Sr}$ and $\mathrm{V}$ concentrations when compared with the Ti-K rich amphiboles. An example of this behaviour can be observed in the zoning profile of Figure 5, where two distinct types of amphiboles (brown and green) have been analysed, with green amphibole being relatively depleted in Ti, K, Sr, Ba and LREE. HFSE and REE also decrease their concentration in amphiboles with lower Ti (Fig. 8a, b, c, d). The amphibole associated with coronitic orthopyroxene does not show a distinct composition with respect to other interstitial amphiboles from La Solanilla. The chondrite-normalised trace element values of amphiboles are characterised by very high REE abundances and negative anomalies at $\mathrm{Eu}$, and the primitive mantle-normalised patterns display negative anomalies at $\mathrm{Pb}, \mathrm{Sr}$ and $\mathrm{Ti}$ (Fig. 8e, f). 
Similarly to other mafic minerals, there is a contrasted trace element composition in phlogopite from both samples, manifested in the high and variable $\mathrm{Cr}$ contents of Talavera (354-1370 ppm), when compared to La Solanilla (3.4-152 ppm) (Table 3). Moreover, phlogopite from this latter gabbro reaches higher $\mathrm{Ba}$ (up to $6430 \mathrm{ppm}$ ), Y (up to $0.28 \mathrm{ppm}$ ) and LREE (e.g. Ce up to $0.4 \mathrm{ppm}$ ) values, and lower $\mathrm{Pb}$ and HFSE contents (e.g., Fig. 9a, b). It is noteworthy the heterogeneity in the phlogopite $\mathrm{Zr}-\mathrm{Hf}$ contents from Talavera (Fig. 9b). Primitive Mantle-normalised patterns of phlogopite are characterised by strong abundances of $\mathrm{Rb}-\mathrm{Ba}, \mathrm{Nb}-\mathrm{Ta}, \mathrm{Pb}$ and $\mathrm{Ti}$, and negative Th-U, LREE and $\mathrm{Y}$ anomalies (Fig. 9c).

\section{Discussion}

\subsection{Constraints on processes during magma crystallization}

With the exception of olivine, all mafic phases present in the La Solanilla sample are characteristically depleted in $\mathrm{Cr}$ with respect to the same minerals in the Talavera gabbro, irrespective of their Mg\# (Table 3; Fig. 6a; Fig. 7a, b, c, d). This fact might be explained by the much higher abundance of Cr-spinel inclusions in olivine from La Solanilla (Fig. 2e), whose crystallization depleted drastically the $\mathrm{Cr}$ content in the residual melt.

Unlike other main minerals, orthopyroxene displays contrasting textural and chemical characteristics in the Talavera and La Solanilla samples. Orthopyroxene from Talavera gabbro yields a negative correlation between incompatible elements and $\mathrm{Mg \#} \mathrm{(e.g.,} \mathrm{Fig.} \mathrm{6c),} \mathrm{coherent} \mathrm{with} \mathrm{a} \mathrm{progressive}$ enrichment of the residual liquid in those elements. On the

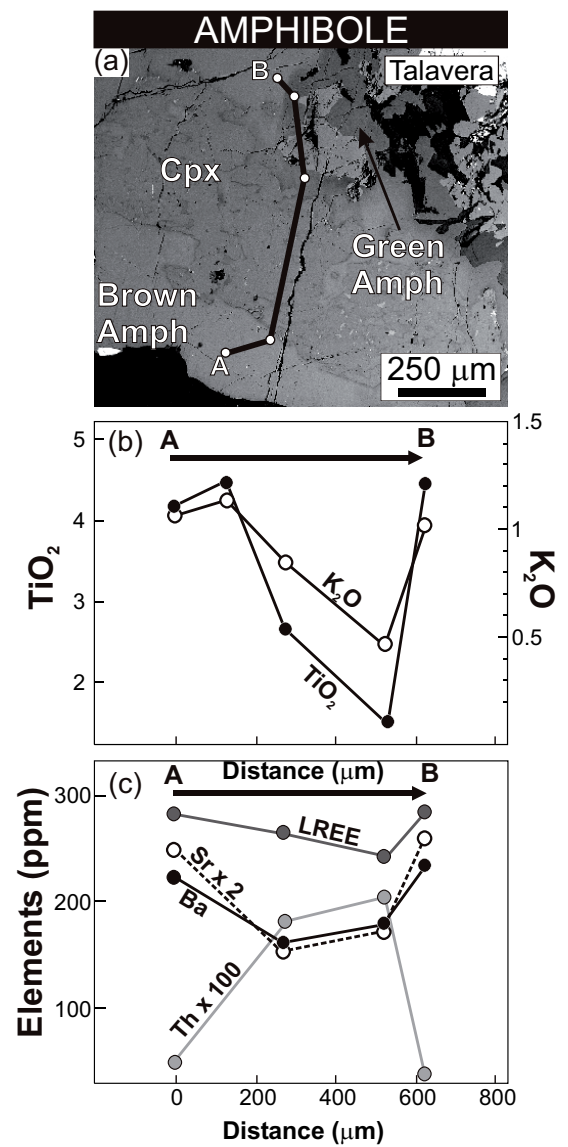

Fig. 5.- $(\mathrm{a}-\mathrm{c})$ Major and trace element zoning profiles within a single amphibole crystal from Talavera sample. The back scattered electron image (a) illustrates the location of the analysis spots (white circles). Major and trace element contents are $\mathrm{wt} \%$ and ppm, respectively. Th and $\mathrm{Sr}$ concentrations are $\mathrm{x} 100$ and $\mathrm{x} 2$, respectively. Note that the image (a) corresponds with Fig. $2 d$
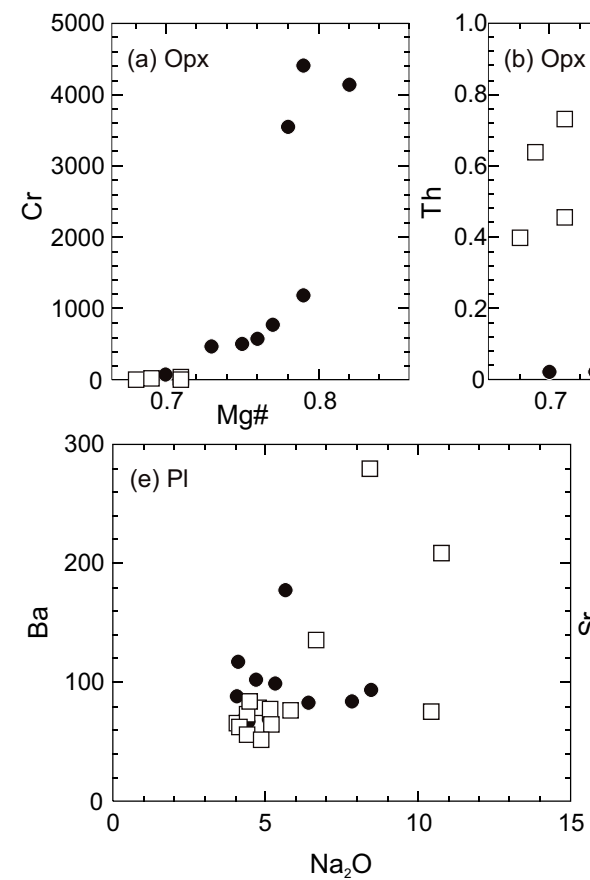
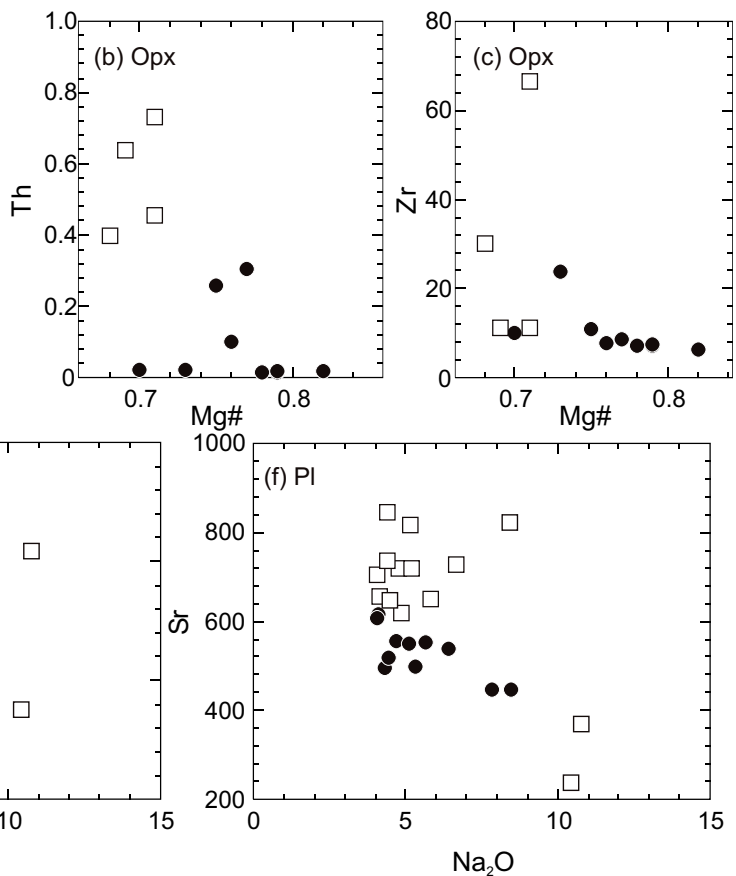

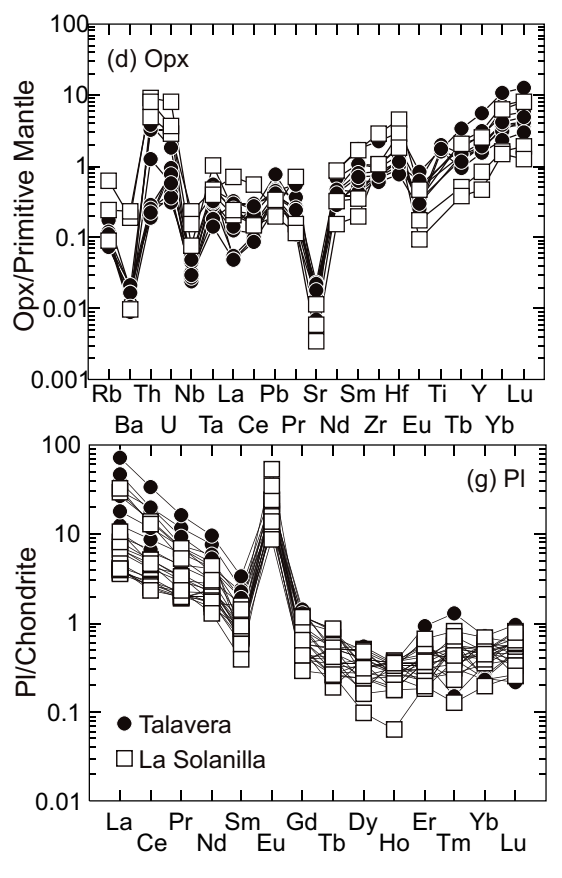

Fig. 6.- Trace element composition (ppm) and Chondrite- or Primitive Mantle-normalised patterns of (a-d) orthopyroxene and (e-g) plagioclase from Talavera and La Solanilla gabbros. Note that trace elements in diagrams a, b, c, e and f have been plotted against a parameter illustrative of the degree of differentiation. Chondrite and Primitive Mantle normalising values after Sun and McDonough (1989) and McDonough and Sun (1995), respectively. 

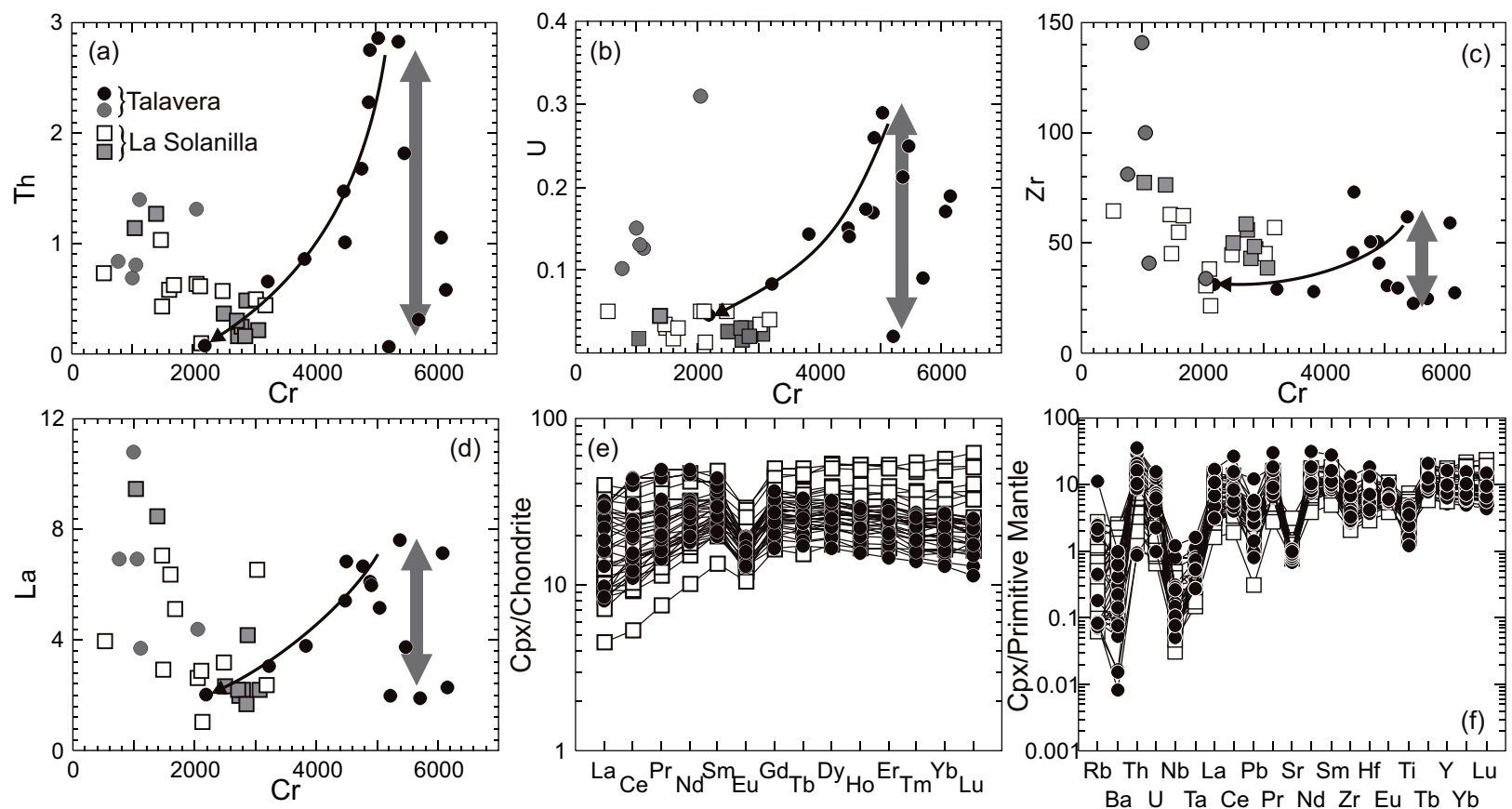

Fig. 7.- Trace element composition ( $\mathrm{ppm})(\mathrm{a}-\mathrm{d})$ and Chondrite- or Primitive Mantle-normalised patterns (e-f) of clinopyroxene from Talavera and La Solanilla gabbros. In diagrams a, b, c and d, the solid black arrow represents the compositional variation trend in Talavera sample, the wide grey double arrow indicates the compositional range for Cr-rich analyses in Talavera gabbro, and the grey filled symbols represent areas partially replaced by amphibole. Note that trace elements in diagrams a, b, $\mathrm{c}$ and d have been plotted against a parameter illustrative of the degree of differentiation. Chondrite and Primitive Mantle normalising values after Sun and McDonough (1989) and McDonough and Sun (1995), respectively.
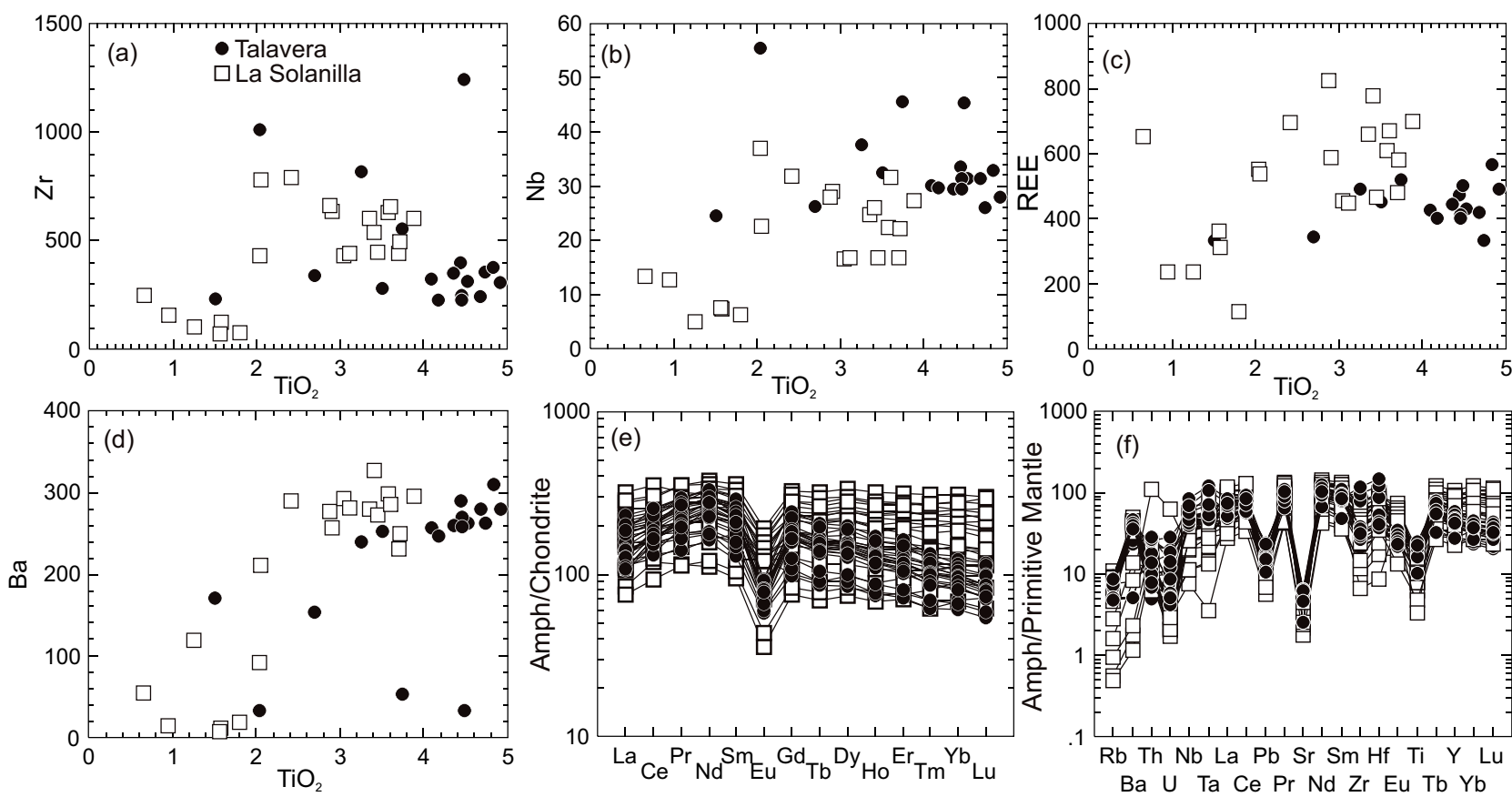

Fig. 8.- Trace element composition (ppm) (a-d) and Chondrite- or Primitive Mantle-normalised patterns (e-f) of amphibole from Talavera and La Solanilla gabbros. Note that trace elements in diagrams a, b, c and d have been plotted against a parameter illustrative of the degree of differentiation. REE in plot c represents the sum of $\mathrm{La}$ to $\mathrm{Lu}$ concentrations (ppm). Chondrite and Primitive Mantle normalising values after Sun and McDonough (1989) and McDonough and Sun (1995), respectively.

other hand, orthopyroxene from La Solanilla, which only appears forming coronas around olivine, have lower $\mathrm{Cr}$ contents and Mg\# values (always bellow 0.74; Fig. 6a) and much higher concentrations for certain incompatible trace elements (e.g., Fig. 6b, c). The presence of composite orthopyroxeneamphibole coronas at olivine-plagioclase contacts (Fig. 2e) has been previously described and attributed to a magmatic origin, rather than metamorphic (e.g. Joesten, 1986; Haas et 
al., 2002). The relatively high concentration of incompatible trace elements (such as LILE and REE) in the coronitic orthopyroxene from La Solanilla (Fig. 6b, c, d) does not favour formation by subsolidus processes, but rather require the involvement of an evolved interstitial silicate liquid in its origin, thus reinforcing the possibility of a late magmatic genesis.

The heterogeneous chemical composition shown by the early-crystallisation phases of the SCS gabbros has been related to some degree of crystal fractionation (Orejana et al., 2009). The continuous normal zoning displayed by plagioclase in the two analysed samples for the present study, characterised by Ab-rich contents towards the rim of idiomorphic prisms (Fig. 4), agrees with the above conclusion. Widespread plagioclase crystallization is also consistent with the negative $\mathrm{Sr}$ and Eu anomalies in the normalised patterns of orthopyroxene, clinopyroxene and amphibole (Fig. 6d; Fig. 7e; Fig. 8e).

On the other hand, chemical zoning in clinopyroxene, either in major or trace elements, is likely influenced by element diffusion during its replacement by amphibole (Fig. 2d, f), which can be observed in both samples (Talavera and La Solanilla). The decrease in $\mathrm{Ti}, \mathrm{Al}$ and $\mathrm{Na}$ (Fig. 4) would be in accordance with their preferential diffusion towards crystallising amphibole, which have higher partition coefficients for these elements. Similar textural and chemical features in clinopyroxene-amphibole reaction areas have been identified in oceanic gabbros by Coogan et al. (2001). Clinopyroxene analyses with the highest $\mathrm{Al}$, Ti and $\mathrm{Na}$ concentrations (and lower Ca) in La Solanilla gabbro (Fig. 3c, d) usually display a low total cation sum ( $97-98 \mathrm{wt} \%)$, likely indicating that they represent secondary highly transformed mineral domains. The fact that clinopyroxene rims are enriched in trace elements more compatible with amphibole (Fig. 4) is likely the result of analysing with a near $50 \mu \mathrm{m}$ diameter laser beam, which has likely ablated part of the $<10 \mu \mathrm{m}$ amphibole micro-inclusions present in clinopyroxene rims associated with reaction zones (Fig. 2f). Due to the uncertainty in the composition of analyses from partially transformed areas (which have been marked in Fig. 3c, d and Fig. 7a, b, c, d), they have not been considered in further discussions.

The above features reveal a complex history of interstitial melt in-situ crystallization. Several textural and chemical observations testify for this process both in the Talavera and La Solanilla gabbroic intrusions. The evidences are: 1) the presence of interstitial minerals rich in highly incompatible trace elements (mainly amphibole, phlogopite, ilmenite, apatite, zircon, baddeleyite and titanite), 2) overgrowth of early crystallization phases towards a more differentiated composition (mainly plagioclase), and 3) disequilibrium between some interstitial crystals and previous minerals (e.g. clinopyroxene-amphibole reaction). Such features have been commonly interpreted as resulting by late-stage crystallization of interstitial melts (e.g. Barnes, 1986; Bédard, 1994; Ross and Elthon, 1997; Tribuzio et al., 1999b). Complete re-equilibration of the early-formed minerals with the residual melt fraction
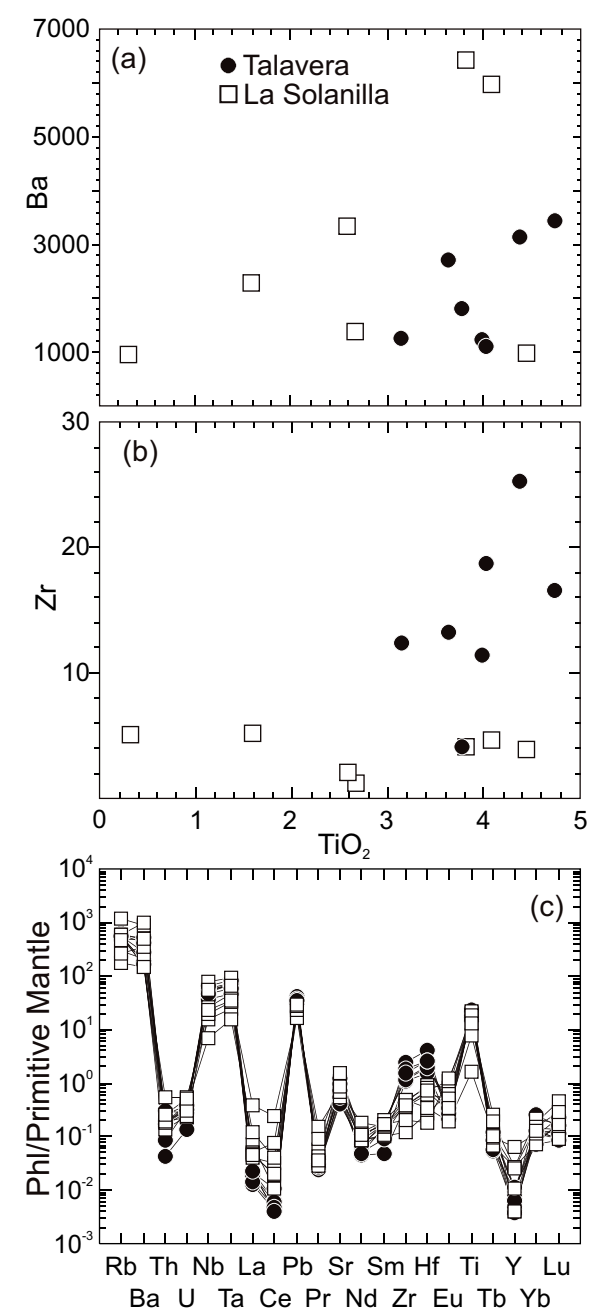

Fig. 9.- Trace element composition (ppm) (a-b) and Primitive Mantle-normalised patterns (c) of phlogopite from Talavera and La Solanilla gabbros. Note that trace elements in diagrams a and $b$ have been plotted against a parameter illustrative of the degree of differentiation. Primitive Mantle normalising values after McDonough and Sun (1995).

was not attained, as demonstrated by the late crystallization of interstitial plagioclase with anorthite contents occasionally below 10, together with the preservation of chemical zoning in this mineral (Fig. 4). The similarity in Mg\# values between the early- and late-crystallized mafic minerals $\left(\mathrm{Mg} \#_{\mathrm{Ol}}=0.78\right.$ $0.63, \mathrm{Mg}_{\mathrm{Opx}}=0.82-0.68, \mathrm{Mg} \#_{\mathrm{Cpx}}=0.84-0.74, \mathrm{Mg} \#_{\mathrm{Amph}}=$ $0.75-0.64, \mathrm{Mg} \#_{\mathrm{Phl}}=0.82-0.56$ ), results somewhat contradictory. However, this feature could be explained in two ways: 1) due to a significant decrease of $\mathrm{Fe}$ in the residual melt (see below the discussion regarding the influence of ilmenite), or 2) advocating a significant increase in the oxygen fugacity, which can induce higher $\mathrm{Mg} \#$ values in hydrous minerals (Scaillet and Evans, 1999). The increment of oxygen fugacity likely occurred in conjunction with the increase of the $\mathrm{H}_{2} \mathrm{O}$ concentration in the late-stage residual melt.

The formation of Ti-rich amphibole in gabbroic rocks has been ascribed in the literature to a metamorphic or magmatic origin. The first possibility has been mainly proposed for solid-state reactions during infiltration of seawater-derived 

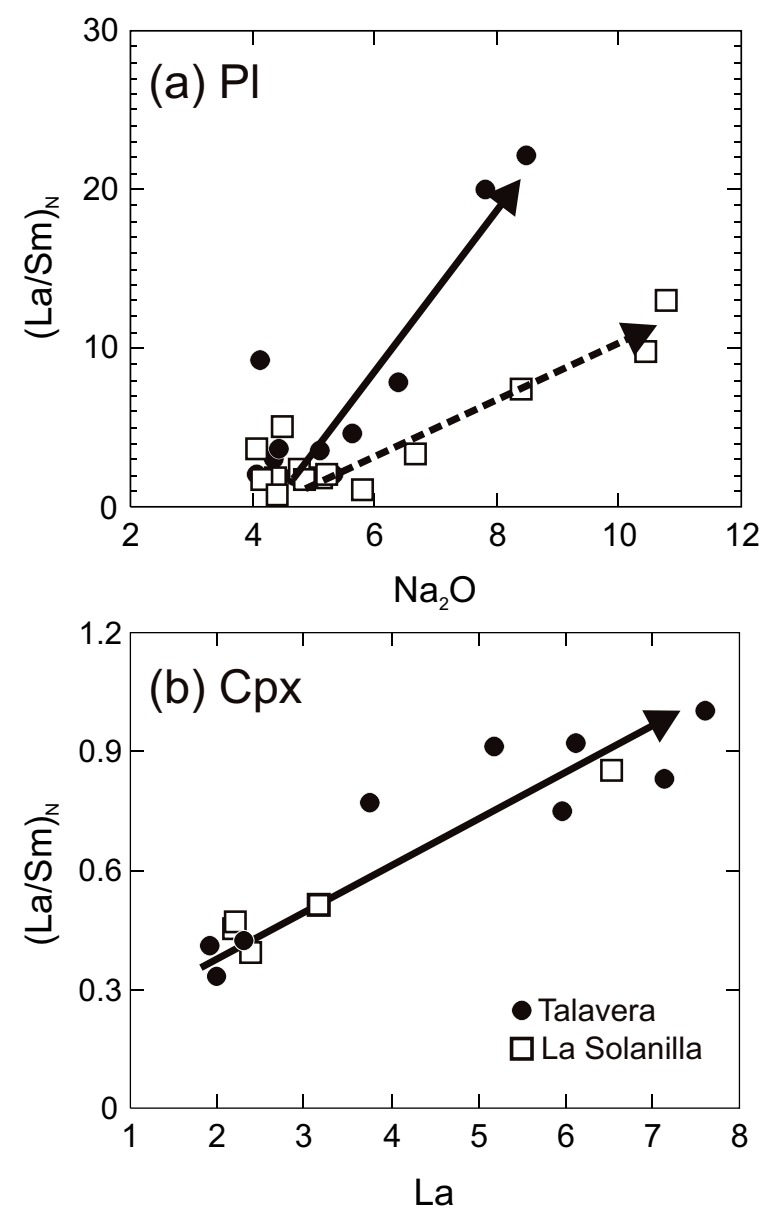

Fig. 10.- (a) $\mathrm{Na}_{2} \mathrm{O}$ vs. $(\mathrm{La} / \mathrm{Sm})_{\mathrm{N}}$ values in plagioclase and (b) La vs. $(\mathrm{La} / \mathrm{Sm})_{N}$ in clinopyroxene from Talavera and La Solanilla gabbros. Only clinopyroxenes with the highest $\mathrm{Cr}$ contents have been plotted in diagram (b). Analyses affected by transformation of clinopyroxene have not been considered. Solid and dotted black arrows represent compositional variation trends. La and Sm concentrations normalised to Chondrite contents (Sun and McDonough, 1989)

fluids through mafic plutonic rocks in oceanic environments (e.g. Coogan et al., 2001 and references therein). Sub-solidus hydrothermal alteration is highly unlikely in the SCS gabbroic bodies considering the temperature estimates ( 825 to $970{ }^{\circ} \mathrm{C}$, hornblende-plagioclase geothermometer; Molina et al., 2009). The very high REE, Y, Zr and Ti concentrations of amphibole from Talavera and La Solanilla intrusions (Fig. $8 \mathrm{a}, \mathrm{e}, \mathrm{f})$, and the fact that amphibole is texturally in equilibrium with other magmatic interstitial phases (e.g. phlogopite, apatite), strongly support its magmatic genesis in the studied samples. The occurrence of Ti-rich amphibole as an interstitial phase in gabbroic rocks, associated with a priori anhydrous incompatible trace element-depleted systems, have been widely explained by late crystallization from more fractionated magmas, after liquidus minerals formation (mostly in tholeiitic basic magmas; e.g. Gillis, 1996; Tribuzio et al., 1999b, 2000; Coogan et al., 2001).

The heterogeneity of clinopyroxene composition in both gabbros might have fairly different origins. As already men- tioned, some analyses from both samples are affected by the replacement of clinopyroxene by amphibole (e.g. see Fig. 3c, d and Fig. 7a, b, c, d), and they are not considered for further discussions. However, a subtle increment in trace element contents towards lower $\mathrm{Cr}$ can be observed in clinopyroxene from the La Solanilla gabbro (Fig. 7a, b, c, d), which points to a progressive enrichment of the residual melt in incompatible elements. This is also suggested by the LREE-enriched nature of interstitial An-poor plagioclase (Fig. 10a). On the contrary, clinopyroxene from the Talavera sample has two contrasting features: 1) extremely variable trace element contents for clinopyroxene with high $\mathrm{Cr}$ values, and 2) decreasing $\mathrm{Ba}$, $\mathrm{Th}, \mathrm{U}, \mathrm{Zr}, \mathrm{Nb}, \mathrm{V}$ and LREE towards lower $\mathrm{Cr}$ concentrations (e.g., Fig. 7a, b, c, d). While the first characteristic will be discussed in more detail in the following section, the depletion of some trace elements in the most evolved clinopyroxene might be interpreted as due to co-precipitation of phases with high partition coefficients for certain elements, such as apatite (LREE), zircon (Zr, Hf, Th, U), baddeleyite (Zr, Hf, U), amphibole $(\mathrm{Nb})$ and phlogopite $(\mathrm{Ba}, \mathrm{Nb}, \mathrm{V})$. The influence of co-crystallising minerals in Talavera can also be identified in the amphibole chemistry. The large brown Ti-K-rich kaersutite likely represents the initial amphibole composition, whereas the lower $\mathrm{Ti}, \mathrm{K}, \mathrm{Rb}, \mathrm{Ba}, \mathrm{Sr}$ and $\mathrm{V}$ contents of the more interstitial green pargasite (Fig. 5) would be caused by coeval crystallization of phlogopite and Ab-rich plagioclase (overgrowths). Nevertheless, the fact that low Ti-K amphibole from Talavera shows an heterogeneous composition for $\mathrm{Zr}-\mathrm{Hf}, \mathrm{Nb}-\mathrm{Ta}$, Th and LREE (e.g. Zr-Nb, Fig. 8a, b), implies that amphibole formation in equilibrium with zircon, baddeleyite, apatite and ilmenite was a local phenomenon. Thus, while phlogopite and interstitial plagioclase seem to have controlled the distribution of LILE and Sr, respectively, in the residual melt, the local precipitation of the above accessory phases have governed HFSE and LREE distribution. More specifically, the relevant role of ilmenite can be also identified in the behavior of $\mathrm{Mg \#}$ and $\mathrm{Ti}$ in amphibole and phlogopite. Both minerals are in equilibrium with ilmenite

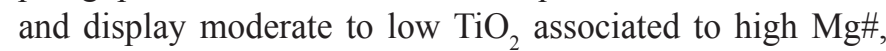
which is an effect that could be expected from the crystallization of a Ti-Fe-rich mineral.

To evaluate more precisely the role played by accessory phases in the trace element distribution we have made a mass balance calculation considering the observed modal proportions of olivine, plagioclase, orthopyroxene, clinopyroxene, amphibole and phlogopite. Several conclusions for both samples can be drawn from the results (see calculation details in caption to Fig. 11): 1) the distribution of most trace elements is controlled by minerals crystallised from the residual interstitial liquid (amphibole, phlogopite and other accessory phases), excepting the HREE, that are significantly incorporated in pyroxene $(\sim 30-50 \%)$, and $\mathrm{Ba}$ and LREE in plagioclase ( 10-30\%); 2) HREE are largely controlled by amphibole, 3) LILE are mainly incorporated into phlogopite $(\mathrm{Rb}$, $\mathrm{Ba}$ ) and plagioclase $(\mathrm{Ba}), 4) \mathrm{Nb}-\mathrm{Ta}$ demonstrate a clear pref- 
erence for amphibole, phlogopite and one accessory mineral (likely ilmenite), and 5) many incompatible trace elements seem to be predominantly controlled by accessory phases present in modal proportion below $1 \%$ : LREE (apatite) and $\mathrm{Th}-\mathrm{U}$ and $\mathrm{Zr}-\mathrm{Hf}$ (zircon and baddeleyite) (Fig. 11).

\subsection{Involvement of an external component}

Although textural and chemical criteria support the precipitation of interstitial minerals from a residual liquid by in-situ crystallization, this process might have progressed either in a closed or in an open system situation.

To discuss these possibilities we have applied the Equilibrium Distribution Method (EDM) of Bédard (1994). This author proposed a mass balance calculation to model the distribution of trace elements between a mineral assemblage and an interstitial trapped melt during crystallization in a closed system, applicable to cumulate rocks or any other solid-liquid system. The equation of Bédard (1994) allows the calculation of any mineral composition if the following variables are known: 1) whole-rock composition, 2) mineral modal proportions, 3) mineral/melt partition coefficients, and 4) trapped melt fraction. We have used this method to test the hypothesis of closed system crystallization by estimating the composition of clinopyroxene, which is the last major phase in equilibrium with the interstitial melt. While whole-rock composition of Talavera and La Solanilla gabbros were determined by Orejana et al. (2009), in this work we have made a detailed estimation of the modal composition in the studied samples (see results in caption to Fig. 11). The mineral/melt partition coefficients used in this study have been extracted from experimental studies on basic magmas at thermodynamic conditions similar to those of the SCS gabbros (see references in caption to Fig. 12).

We have considered a $20 \%$ residual melt fraction, which represents a proportion coherent with the modal amount of minerals crystallised directly from the interstitial liquid. The modelled clinopyroxene composition is compared in Fig. 12 with the most primitive clinopyroxene from each sample (highest $\mathrm{Cr}$ contents). The pattern predicted by the equation of Bédard (1994) matches the composition of clinopyroxene from each sample, excepting LILE and LREE whose concentrations plot below or towards the lowest values of the represented clinopyroxene range (Fig. 12). These differences in LILE and LREE contents are difficult to reconcile with a closed magmatic system. The extremely high concentrations of elements such as Th and $\mathrm{U}$ in clinopyroxene (up to $2.86 \mathrm{ppm}$ and $0.3 \mathrm{ppm}$ in the Talavera gabbro; Fig. 7a, b) would imply dozens to hundreds ppm of Th in the liquid, even considering a wide range of mineral/basic melt partition coefficients (e.g. 0.06 to 0.01; Lemarchand et al., 1987; Hauri et al., 1994, respectively). Moreover, other minerals display remarkably high trace element concentrations (mainly REE), such as Na-rich plagioclase (Fig. 6g) and amphibole (Fig. 8e, f).
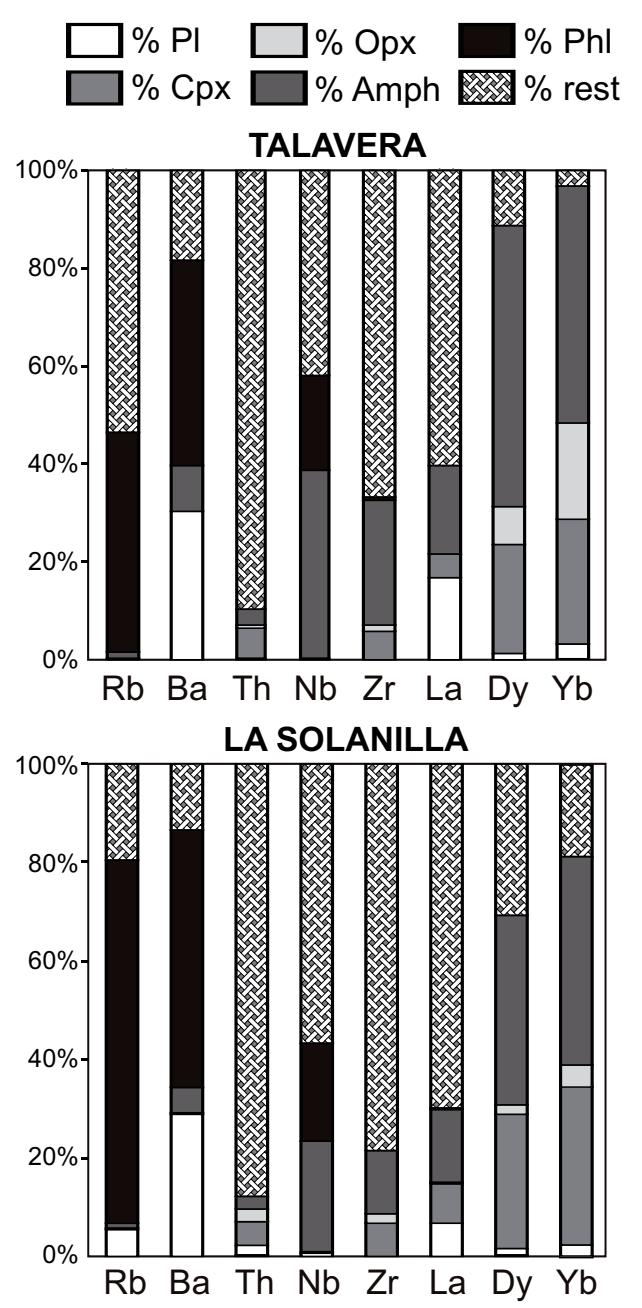

Fig. 11.- Bar diagram showing the relative proportion of selected trace elements in the main minerals from the Talavera and $\mathrm{La}$ Solanilla gabbros obtained by a mass balance calculation. Trace element composition of minerals used in the calculation considers averaged values (partially transformed clinopyroxene have not been considered). Modal composition used are those of Table 1: ol (7\%), pl (54\%), opx (16\%), cpx (12\%), amph (6\%), phl $(3 \%)$ and rest (2\%) for Talavera sample and ol (15\%), pl (58\%), opx $(6 \%)$, cpx (14\%), amph (3\%), phl (3\%) and rest $(1 \%)$ for La Solanilla sample

LILE and LREE enrichment in minerals (mainly clinopyroxene) from gabbroic rocks have been related with a process of contamination by an external evolved liquid (e.g. Tribuzio et al., 1999a; Tiepolo and Tribuzio, 2005; Renna et al., 2006). Figure 10b illustrate that the enrichment of the most incompatible REE in clinopyroxene (e.g., La) is associated to a higher degree of fractionation of LREE. High $(\mathrm{La} / \mathrm{Sm})_{\mathrm{N}}$ values also correlate positively with $\mathrm{Na}_{2} \mathrm{O}$ in plagioclase (Fig. 10a). This behaviour might be explained advocating the involvement of a LREE-rich external fluid/ melt. A recent study by Villaseca et al. (2011) focused on the geochronology and Hf isotope composition of zircons from the SCS gabbros, highlighted the heterogeneous \&Hf values (+2.9 to -1.1$)$ displayed by magmatic zircons from the Talavera gabbro and the presence of xenocrystic zircons with $\varepsilon \mathrm{Hf}$ 
values typical of crustal rocks ( -3 to -9$)$ in the La Solanilla gabbro. These zircon data clearly support that both gabbroic bodies were contaminated to some degree either by partial melts derived from local assimilation of metamorphic wallrocks or due to hybridization with coeval Variscan granite magmas as most studies suggest (e.g., Bea et al., 1999; Villaseca et al., 2011).

\subsection{Nature of the parental magmas}

Magmatism in collisional orogens is dominated by complex processes which commonly involve melting of contrasted sources (crust and mantle), fractionation, mixing of the generated magmas and local assimilation of country rocks. Furthermore, hybridization may occur at different crustal levels. Thus, the geochemical affinity of magmas parental to mantle-derived rocks is not always a straightforward issue.

The late-Variscan mafic rocks from central Spain develop well-characterised medium- to high-K calc-alkaline trends (Villaseca et al., 2004; Orejana et al., 2009; Scarrow et al., 2009), with the most primitive gabbros showing orthopyroxene (typically subalkaline) as a near liquidus phase. However, the presence of Ti-rich minerals in these rocks, such as kaersutite and Ti-phlogopite, have suggested an alkaline affinity (Molina et al., 2009), and the possibility that the calc-alkaline composition resulted from chemical interaction (hybridization) at depth between alkaline basic magmas and felsic melts, prior to the crystallization process (Scarrow et al., 2009). Kaersutite and pargasite are amphiboles typical of alkaline basic rocks and metasomatised mantle xenoliths and their formation occurs early during the crystallization process (e.g. Martin, 2007). Nevertheless, the presence of these minerals in subalkaline series is not an uncommon phenomenon. Féménias et al. (2006) described core to rim zoning patterns in amphiboles from basaltic to rhyolitic calc-alkaline dykes, including pargasite, kaersutite, edenite and magnesiohastingsite cores. A similar chemical evolution was found by Kawakatsu and Yamaguchi (1987) from brown magnesiohornblende and magnesiohastingsite towards green magnesiohorblende and actinolite in calc-alkaline plutonic rocks. In fact, pargasite displays a heterogeneous major element composition, with pargasite s.s. occurring almost exclusively in alkaline rocks and the tschermakitic pargasite being typical of calc-alkaline series (Ridolfi and Renzulli, 2012). The composition of pargasite from the Talavera and La Solanilla gabbros is similar to the tschermakitic pargasite described by Ridolfi and Renzulli (2012) and fits the chemical criteria proposed by these authors (mainly $\mathrm{K}$ in A-site below 0.22 apfu) for amphiboles formed from calc-alkaline magmas. Even kaersutite can precipitate as an interstitial mineral from tholeiitic magmas (e.g. Tribuzio et al., 2000; Coogan et al., 2001). These latter authors suggest that amphibole formation can be ascribed to the percolation of relatively evolved melts. Ti-rich biotite-phlogopite has also been described in subalkaline basic plutonic rocks (e.g., Tribuzio et al., 1999a; Costa

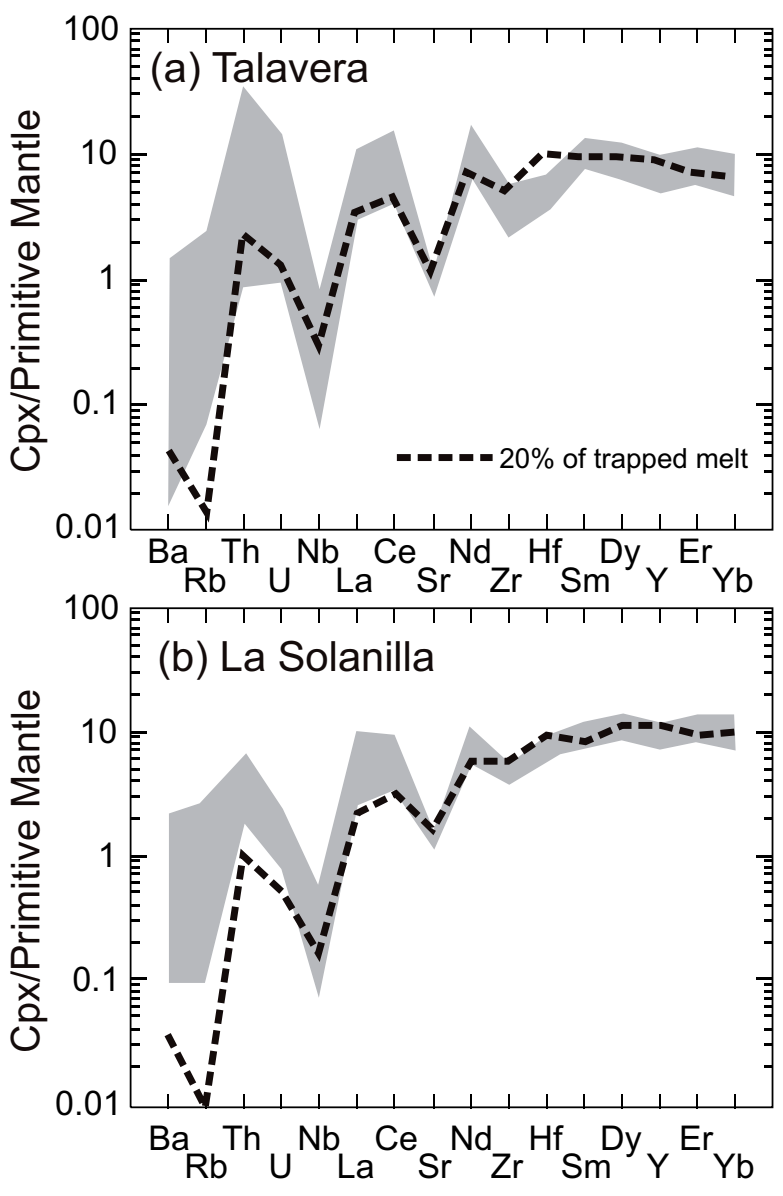

Fig. 12.- Primitive Mantle-normalised trace element abundances of clinopyroxenes calculated with the EDM method (Equilibrium Distribution Method; from Bédard, 1994) for (a) Talavera and (b) La Solanilla gabbros, assuming a trap melt fraction (TMF) of $20 \%$. Whole rock composition extracted from Orejana et al. (2009). Modal composition as that of caption to Fig. 11, with the exception of clinopyroxene which has been balanced with the TMF in order to get a total of $100 \%$. Partition coefficients used: olivine/melt from Bédard (1994); plagioclase/melt from Bindeman and Davis (2000) and Bédard (1994); ortopyroxene/melt from Green et al. (2000) and Kennedy et al. (1993) and clinopyroxene/melt from Hart and Dunn (1993), Hauri et al. (1994) and Adam and Green (2006). Trace element abundances of Cr-rich clinopyroxenes from each sample (grey field) are also reported for comparison. Normalising values after McDonough and Sun (1995).

et al., 2002; Claeson and Meurer, 2004). Thus, the presence of Ti-rich minerals can not be considered as an unambiguous evidence of crystallization from an alkaline melt.

Amphibole and phlogopite in the Talavera and La Solanilla gabbros are not abundant early precipitated phases, but latestage interstitial minerals present in low proportions $(<9 \%)$, locally in disequilibrium with the surrounding phases (e.g. clinopyroxene; Fig. 2d, f). These textural and chemical features, taken together with the whole-rock chemistry, support a subalkaline affinity for the SCS Variscan basic melts. We propose that the extensive crystallization of initially $\mathrm{H}_{2} \mathrm{O}$ subsaturated magmas, in conjunction with the addition of 
external components during emplacement, might have led to subsequent enrichment in incompatible elements, including volatiles, and melt saturation in amphibole. The highly enriched trace element composition of most minerals in the studied gabbros (e.g. LREE in plagioclase, REE in orthopyroxene, clinopyroxene and amphibole) exclude the possibility of an initial tholeiitic magma, and are very similar to the composition of clinopyroxene and amphibole in other calcalkaline post-orogenic Variscan gabbros (e.g. the CorsicaSardinia batholith; Renna et al., 2006).

Apart from the late growth of Ti-rich minerals, no strong evidence against a dominant calc-alkaline affinity in the SCS gabbros is apparent. The last events of the Variscan orogeny are characterised in central Spain (and in many other western Variscan massifs) by a widespread felsic magmatism, with abundant granite intrusions emplaced in the range $\sim 310-298$ Ma (e.g., Orejana et al., 2012; Merino Martínez et al., 2014, and references therein). Late Variscan magmatism, including both the felsic magmas and the accompanying scarce basic melts, could have been originated by adiabatic decompression as lithosphere experienced considerable thinning during the orogen collapse (e.g., Orejana et al., 2009; Scarrow et al., 2009). Whole-rock geochemistry of the primitive olivinebearing gabbros, such as negative $\mathrm{Nb}-\mathrm{Ta}$ and $\mathrm{Ti}$ anomalies, LILE enrichment and low $\mathrm{Ta} / \mathrm{Yb}$ and $\mathrm{Ce} / \mathrm{Pb}$ ratios, points to a calc-alkaline nature (Orejana et al., 2009). Furthermore, other basic rocks that intruded as large dyke swarms in the SCS in a later stage ( $290 \mathrm{Ma}$; Galindo et al., 1994), show similar calc-alkaline fingerprints as those described above (Gb2 and Gb3 series in Villaseca et al., 2004). Accordingly, we think that a lithospheric mantle metasomatised by crustallike (collisional?) components would be the source of these post-collisional basic magmas.

The involvement of an alkaline component in the origin of the SCS primitive gabbros is not obvious. Their whole-rock composition is difficult to reconcile with a possible magma mixing process involving an alkaline basic melt and a felsic component. The concentration of some major and trace elements in the SCS gabbros do not plot in an intermediate position between that of a possible felsic melt (e.g., the SCS felsic Variscan magmatism) hybridized with an alkaline basic magma. Recent studies have highlighted the importance of crystal transfer as a mechanism capable of producing non-linear elemental correlations between felsic and mafic magmas (e.g., Ubide et al., 2014). However, the SCS gabbros display lower or overlapping contents in some minor and trace elements (Ti, Nb, Zr) with respect to granite composition. This feature implies a notable deviation from an alkaline composition and, accordingly, would mean a significant crystal transfer, if this process had been important during mixing. The primitive nature of the analysed olivine-bearing gabbros, together with the absence of felsic minerals showing disequilibrium textures, prevents significant mixing with coeval granite magmas and supports that the calc-alkaline signature in the SCS gabbros could be considered as a primary feature.
The only basic alkaline magmatic rocks outcropping in the SCS are lamprophyric and diabasic dykes (Orejana et al., 2008) of Permian age (ca. 264 Ma; e.g., Scarrow et al., 2006; Perini and Timmerman, 2008), which intrude about $35 \mathrm{Ma}$ after the Variscan gabbros are emplaced (305-300 Ma; Villaseca et al., 2011), in a fairly different tectonic regime. Some of these alkaline dykes display isotopically depleted signatures ( $\varepsilon \mathrm{Nd} \sim+7$; Orejana et al., 2008), which suggests that metasomatism in the source mantle likely occurred shortly before alkaline melt generation (Orejana et al., 2008). The Variscan basic magmatism at ca. $300 \mathrm{Ma}$ would register a previous and different mantle source. This transformation of the lithospheric (and asthenospheric) mantle under central Spain has been associated with delamination of dense crustal blocks in the thickened Variscan collisional belt, which is supported by $\mathrm{Sm}-\mathrm{Nd}$ isotopic signatures of pre-Variscan, Variscan and post-Variscan basic magmas (e.g., Villaseca et al., 2004; Orejana et al., 2008; Gutierrez-Alonso et al., 2011).

\section{Conclusions}

The Talavera and La Solanilla gabbroic intrusions display a heterogeneous mineral composition, including incompatible trace element-rich interstitial phases, such as amphibole, phlogopite and other accessory minerals (ilmenite, zircon, apatite, baddeleyite, titanite). Major element composition of the main minerals show a high degree of variation: for instance, plagioclase show zoning patterns ranging from $\mathrm{Ca}-$ rich cores to Na-rich overgrowths and clinopyroxene zoning seems to be related to disequilibrium reactions, leading to replacement by amphibole at crystal rims.

Trace element contents in clinopyroxene from both gabbros towards a more evolved composition accounts for a progressive enrichment of the residual melt in incompatible elements. However, this tendency is clearly modified by coprecipitation of phases with high partition coefficients for specific elements, such as apatite (LREE), zircon (Zr, Hf, Th, $\mathrm{U})$, baddeleyite ( $\mathrm{Zr}, \mathrm{Hf}, \mathrm{U})$, amphibole $(\mathrm{Nb})$ and phlogopite $(\mathrm{Ba}, \mathrm{Nb}, \mathrm{V})$. A simple mass balance calculation indicates that the distribution of most trace elements is controlled by minerals crystallised from the interstitial liquid (amphibole, phlogopite and accessory phases).

The use of the Equilibrium Distribution Method of Bédard (1994) highlights that the very high LILE and LREE contents in clinopyroxene from both gabbroic samples can not be explained exclusively by an in-situ crystallization process in a closed-system situation. Positive correlation between $\mathrm{La} / \mathrm{Sm}$ normalised ratios and $\mathrm{La}$ and $\mathrm{Na}$ contents, in clinopyroxene and plagioclase respectively, serve as evidence of contamination with an external evolved liquid, likely associated with granite melt hybridization, as stated by previous isotopic studies.

Petrographic features (e.g., presence of orthopyroxene, late precipitation of amphibole) and mineral composition (e.g., trace element-enriched clinopyroxene and amphibole com- 
position) in the primitive SCS gabbros, seem to favour an original calc-alkaline geochemical affinity, instead of alkaline or tholeiitic. The bulk rock composition of the SCS gabbros and possible felsic and basic alkaline magmas go against a possible mixing process in their origin. Available isotopic data from the literature also suggest that mantle metasomatism beneath central Spain, before the end of the Variscan orogeny, is more likely caused by continental crust components, probably associated with delamination of dense crustal blocks from the thickened Variscan collisional belt.

\section{Acknowledgements}

We acknowledge Alfredo Fernández Larios and José González del Tánago for their assistance with the electron microprobe analyses in the Centro Nacional de Microscopía Electrónica (UCM). The valuable and constructive reviews by Dra. Teresa Ubide and an anonymous reviewer, together with the editorial handling of Dr. José López-Gómez, helped us to improve the initial version of the manuscript. Cecilia Pérez-Soba is thanked for her assistance during laser data acquisition and also for useful comments which helped to improve the final manuscript. The SYNTHESYS European Union-funded Integrated Infrastructure Initiative grant has allowed us to carry out the laser ICP-MS mineral analyses at the Natural History Museum of London. This work is included in the objectives of, and supported by, the CGL201232822 project of the Ministerio de Economía y Competitividad of Spain and the 910492 UCM research group.

\section{References}

Adam, J., Green, T. (2006): Trace element partitioning between micaand amphibole-bearing garnet lherzolite and hydrous basanitic melt: 1. Experimental results and the investigation of controls on partitioning behavior. Contributions to Mineralogy and Petrology 152, 1-17. doi: 10.1007/s00410-006-0085-4

Barnes, S.J. (1986): The effect of trapped liquid crystallization on cumulus mineral compositions in layered intrusions. Contributions to Mineralogy and Petrology 93, 524-531. doi: 10.1007/BF00371722

Bea, F., Montero, P., Molina, J.F. (1999): Mafic precursors, peraluminous granitoids, and late lamprophyres in the Avila batholith; a model for the generation of Variscan batholiths in Iberia. Journal of Geology 107, 399-419. doi: 10.1086/314356

Bea, F., Montero, P., Zinger, T. (2003): The nature, origin, and thermal influence of the granite source layer of Central Iberia. Journal of Geology 111, 579-595. doi: 10.1086/376767

Bédard, J.H. (1994): A procedure for calculating the equilibrium distribution of trace elements among the minerals of cumulate rocks, and the concentrations of trace elements in the coexisting liquids. Chemical Geology 118, 143-153. doi: 10.1016/0009-2541(94)90173-2

Bernstein, S. (2006): In situ fractional crystallization of a mafic pluton: Microanalytical study of a Palaeogene gabbronorite plug in East Greenland. Lithos 92, 222-237. doi: 10.1016/j.lithos.2006.03.034

Bindeman, I.N., Davis, A.M. (2000): Trace element partitioning between plagioclase and melt: investigation of dopant influence on partition behavior. Geochimica et Cosmochimica Acta 64, 2863-2878. doi: 10.1016/S0016-7037(00)00389-6

Borghini, G., Rampone, E. (2007): Postcumulus processes in oceanic- type olivine-rich cumulates: the role of trapped melt crystallization versus melt/rock interaction. Contributions to Mineralogy and Petrology 154, 619-633. doi: 10.1007/s00410-007-0217-5

Castro, A., Patiño Douce, A.E., Corretgé, L.G., De la Rosa, J.D., ElBiad, M., El-Hmidi, H. (1999): Origin of peraluminous granites and granodiorites, Iberian Massif, Spain. An experimental test to granite petrogenesis. Contributions to Mineralogy and Petrology 135, 255276. doi: 10.1007/s004100050511

Cawthorn, R.G., Sander, B.K., Jones, I.M. (1992): Evidence for the trapped liquid shift effect in the Mount Ayliff intrusion, South Africa. Contributions to Mineralogy and Petrology 111, 194-202. doi: 10.1007/BF00348951

Cebriá, J.M., López-Ruiz, J., Doblas, M., Martins, L.T., Munha, J. (2003): Geochemistry of the Early Jurassic Messejana-Plasencia dyke (Portugal-Spain); Implications on the Origin of the Central Atlantic Magmatic Province. Journal of Petrology 44, 547-568. doi: 10.1093/petrology/44.3.547

Charlier, B., Vander Auwera, J., Duchesne, J-C. (2005): Geochemistry of cumulates from the Bjerkreim-Sokndal layered intrusion (S. Norway) part II. REE and the trapped liquid fraction. Lithos 83, 255-276. doi: 10.1016/j.lithos.2005.03.005

Claeson, D.T., Meurer, W.P. (2004): Fractional crystallization of hydrous basaltic "arc-type" magmas and the formation of amphibole-bearing gabbroic cumulates. Contributions to Mineralogy and Petrology 147, 288-304. doi: 10.1007/s00410-003-0536-0

Coogan, L.A., Wilson, R.N., Gillis, K.M., MacLeod, C.J. (2001): Nearsolidus evolution of oceanic gabbros: Insights from amphibole geochemistry. Geochimica et Cosmochimica Acta 65, 4339-4357. doi: 10.1016/S0016-7037(01)00714-1

Costa, F., Dungan, M.A., Singer, B.S. (2002): Hornblende- and phlogopite-bearing gabbroic xenoliths from volcán San Pedro $\left(36^{\circ} \mathrm{S}\right)$, Chilean Andes: evidence for melt and fluid migration and reactions in subduction-related plutons. Journal of Petrology 43, 219-241. doi: 10.1093/petrology/43.2.219

Dias, G., Leterrier, J. (1994): The genesis of felsic-mafic plutonic associations: a Sr and $\mathrm{Nd}$ isotopic study of the Hercynian Braga Granitoid Massif (Northern Portugal). Lithos 32, 207-223. doi: 10.1016/00244937(94)90040-X

Díaz-Alvarado, J., Castro, A., Fernández, C., Moreno-Ventas, I. (2011): Assessing bulk assimilation in cordierite-bearing granitoids from the Central System Batholith, Spain; experimental, geochemical and geochronological constraints. Journal of Petrology 52, 223-256. doi: 10.1093/petrology/egq078

Féménias, O., Mercier, J.C.C, Nkono, C., Diot, H., Berza, T., Tatu, M., Demaiffe, D. (2006): Calcic amphibole growth in calc-alkaline magmas: evidence from the Motru dike swarm (southern Carpathians, Romania). American Mineralogist 91, 73-81. doi: 10.2138/ am.2006.1869

Fernández-Suárez, J., Arenas, R., Jeffries, T.E., Whitehouse, M.J., Villaseca, C. (2006): A U-Pb study of zircons from a lower crustal granulite xenolith of the Spanish Central system: a record of Iberian lithospheric evolution from the Neoproterozoic to the Triassic. Journal of Geology 114, 471-483. doi: 10.1086/504180

Franco, M.P., García de Figuerola, L.C. (1986): Las rocas básicas y ultrabásicas en el extremo occidental de la Sierra de Ávila. Studia Geologica Salmanticencia 23, 193-219.

Franco, M.P., Sánchez-García, T. (1987): Características petrológicas del área de El Mirón. In: F. Bea, A. Carnicero, J.C. Gonzalo, M. López Plaza, M.D. Rodríguez Alonso (eds), Geología de los granitoides y rocas asociadas del Macizo Hespérico. Rueda, Madrid. pp. 293-313.

Galán, G., Pin, C., Duthou, J.L. (1996): Sr-Nd isotopic record of multistage interactions between mantle-derived magmas and crustal components in a collision context: The ultramafic-granitoid association from Vivero (Hercynian belt, NW Spain). Chemical Geology 131, 
67-91. doi: 10.1016/0009-2541(96)00027-7

Galindo, C., Huertas, M.J., Casquet, C. (1994): Cronología Rb-Sr y KAr de diques de la Sierra de Guadarrama (Sistema Central Español). Geogaceta 16, 23-26.

Gao, Y., Hoefs, J., Hellebrand, E., Von Der Handt, A., Snow, J.E. (2007): Trace element zoning in pyroxenes from ODP Hole 735B gabbros: diffusive exchange or synkinematic crystal fractionation? Contributions to Mineralogy and Petrology 153, 429-442. doi: 10.1007/ s00410-006-0158-4

Gillis, K.M. (1996): Rare earth element constraints on the origin of amphibole in gabbroic rocks from Site 894, Hess Deep. In: C. Mével, K.M. Gillis, J.F. Allan, P.S. Meyer (eds.), Proceedings of the Ocean Drilling Program, scientific results. Collage Station, TX, pp. 59-75.

Green, T.H., Blundy, J.D., Adam, J., Yaxley, G.M. (2000): SIMS determination of trace element partition coefficients between garnet, clinopyroxene and hydrous basaltic liquids at 2-7.5 GPa and 1080 1200 C. Lithos 53, 165-187. doi: 10.1016/S0024-4937(00)00023-2

Gutiérrez-Alonso, G., Murphy, J.B., Fernández-Suárez, J., Weil, A.B., Franco, M.P., Gonzalo, J.C. (2011): Lithospheric delamination in the core of Pangea: Sm-Nd insights from the Iberian mantle. Geology 39, 155-158. doi: 10.1130/G31468.1

Haas, G.J.L.M., Nijland, T.G., Valbracht, P.J., Maijer, C., Verschure, R., Andersen, T. (2002): Magmatic versus metamorphic origin of olivine-plagioclase coronas. Contributions to Mineralogy and Petrology 143, 537-550. doi: 10.1007/s00410-002-0362-9

Hart, S.R., Dunn, T. (1993): Experimental cpx/melt partitioning of 24 trace elements. Contributions to Mineralogy and Petrology 113, 1-8. doi: 10.1007/BF00320827

Hauri, E.H., Wagner, T.P., Grove, T.L. (1994): Experimental and natural partitioning of $\mathrm{Th}, \mathrm{U}, \mathrm{Pb}$ and other trace elements between garnet, clinopyroxene and basaltic melts. Chemical Geology 117, 149-166. doi: 10.1016/0009-2541(94)90126-0

Joesten, R. (1986): The role of magmatic reaction, diffusion and annealing in the evolution of coronitic microstructure in troctolitic gabbro from Risør, Norway. Mineralogical Magazine 50, 441-467. doi: 10.1180/minmag. 1986.050.357.08

Kawakatsu, K., Yamaguchi, Y. (1987): Successive zoning in amphiboles during progressive oxidation in the Daito-Yokota granitic complex, San-in belt, southwest Japan. Geochimica et Cosmochimica Acta 51, 535-540. doi: 10.1016/0016-7037(87)90067-6

Kennedy, A.K., Lofgren, G.E., Wasserburg, G.J. (1993): An experimental study of trace element partitioning between olivine, orthopyroxene and melt in chondrules: equilibrium values and kinetic effects. Earth and Planetary Science Letters 115, 177-195. doi: 0.1016/0012-821X(93)90221-T

Leake, B.E., Woolley, A.R., Arps, C.E.S., Birch, W.D., Gilbert, M.C., Grice, J.D., Hawthorne, F.C., Kato, A., Kisch, H.J., Krivovichev, V.G., Linthout, K., Laird, J, Mandarino, J.A., Maresch, W.V., Nickel, E.H., Rock, N.M.S., Schumacher, J.C., Smith, D.C., Stephenson, N.C.N., Ungaretti, L., Whittaker, E.J.W., Youzhi, G., (2004): Nomenclature of amphiboles; report of the subcommittee on amphiboles of the International Mineralogical Association, Commission on New Minerals and Mineral Names. Canadian Mineralogist 35, 219-246.

Lemarchand, F., Benoit, V., Calais, G. (1987): Trace element distribution coefficients in alkaline series. Geochimica et Cosmochimica Acta 51, 1071-1081. doi: 10.1016/0016-7037(87)90201-8

López-Moro, F.J., López-Plaza, M., (2004): Monzonitic series from the Variscan Tormes Dome (Central Iberian Zone): petrogenetic evolution from monzogabbro to granite magmas. Lithos 72, 19-44. doi: 10.1016/j.lithos.2003.08.002

Martin, R.F., (2007): Amphibole in the igneous environment. Reviews in Mineralogy and Geochemistry 67, 323-358. doi: 10.2138/ rmg.2007.67.9

McDonough, W.F., Sun, S.S. (1995): The composition of the Earth.
Chemical Geology 120, 223-253. doi: 10.1016/0009-2541(94)00140-4

Merino Martínez, E., Villaseca, C., Orejana, D., Pérez-Soba, C., Belousova, E., Andersen, T. (2014): Tracing magma sources of three different S-type peraluminous granitoid series by in situ U-Pb geochronology and Hf isotope zircon composition: The Variscan Montes de Toledo batholith (central Spain). Lithos 200-201, 273-298. doi: 10.1016/j.lithos.2014.04.013

Molina, J.F., Scarrow, J.H., Montero, P.G., Bea, F. (2009): High-Ti amphibole as a petrogenetic indicator of magma chemistry: evidence for mildly alkalic-hybrid melts during evolution of Variscan basic-ultrabasic magmatism of Central Iberia. Contributions to Mineralogy and Petrology 158, 69-98. doi: 0.1007/s00410-008-0371-4

Molina, J.F., Montero, P.G., Bea, F., Scarrow, J.H. (2012): Anomalous xenocryst dispersion during tonalite-granodiorite crystal mush hybridization in the mid crust: Mineralogical and geochemical evidence from Variscan appinites (Avila Batholith, Central Iberia). Lithos 153, 224-242. doi: 10.1016/j.lithos.2012.03.021

Monjoie, P., Bussy, F., Lapierre, H., Pfeifer, H.R. (2005): Modeling of in-situ crystallization processes in the Permian mafic layered intrusion of Mont Collon (Dent Blanche nappe, Western Alps). Lithos 83, 317-346. doi: 10.1016/j.lithos.2005.03.008

Moreno-Ventas, I., Rogers, G., Castro, A. (1995): The role of hybridization in the genesis of the Hercynian granitoids in the Gredos Massif, Spain: inferences from $\mathrm{Sr}-\mathrm{Nd}$ isotopes. Contributions to Mineralogy and Petrology 120, 137-149. doi: 10.1007/BF00287111

Natland, J.H., Dick, H.J.B. (2001): Formation of the lower ocean crust and the crystallization of gabbroic cumulates at a very slowly spreading ridge. Journal of Volcanology and Geothermal Research 110, 191-233. doi: 10.1016/S0377-0273(01)00211-6

Orejana, D., Villaseca, C., Billström, K., Paterson, B.A. (2008): Petrogenesis of Permian alkaline lamprophyres and diabases from the Spanish Central System and their geodynamic context within western Europe. Contributions to Mineralogy and Petrology 156, 477-500. doi: 10.1007/s00410-008-0297-x

Orejana, D., Villaseca, C., Pérez-Soba, C., López-García, J.A., Billström, K. (2009): The Variscan gabbros from the Spanish Central System: A case for crustal recycling in the sub-continental lithospheric mantle? Lithos 110, 262-276. doi: 10.1016/j.lithos.2009.01.003

Orejana, D., Villaseca, C., Armstrong, R.A., Jeffries, T.E. (2011): Geochronology and trace element chemistry of zircon and garnet from granulite xenoliths: constraints on the tectonothermal evolution of the lower crust under central Spain. Lithos 124, 103-116. doi: 10.1016/j. lithos.2010.10.011

Orejana, D., Villaseca, C., Valverde-Vaquero, P., Belousova, E.A., Armstrong, R.A. (2012): U-Pb geochronology and zircon composition of late Variscan S- and I-type granitoids from the Spanish Central System batholith. International Journal of Earth Sciences 101, 1789-1815. doi:10.1007/s00531-012-0750-y. doi: 10.1007/s00531-012-0750-y

Pearce, N.J.G., Perkins, W.T., Westgate, J.A., Gorton, M.P., Jackson, S.E., Neal, C.R., Chenery, S.P. (1997): A compilation of new and published major and trace element data for NIST SRM 610 and NIST SRM 612 glass reference materials. Geostandards Newsletter 21, 115-144. doi: 10.1111/j.1751-908X.1997.tb00538.x

Perini, G., Timmerman, M.J. (2008): Permian ${ }^{40} \mathrm{Ar} /{ }^{39} \mathrm{Ar}$ ages for postVariscan minor intrusions in the Iberian Range and Spanish Central System. Geologica Acta 6, 335-344.

Pinarelli, L., Rottura, A. (1995): Sr and Nd isotopic study and Rb-Sr geochronology of the Béjar granites, Iberian Massif, Spain. European Journal of Mineralogy 7, 577-589. doi: 10.1127/ejm/7/3/0577

Recio, C., Fallick, A.E., Ugidos, J.M. (1992): A stable isotopic $\left(\delta^{18} \mathrm{O}\right.$, $\delta \mathrm{D})$ study of the late-Hercynian granites and their host-rocks in the Central Iberian Massif (spain). Transactions of the Royal Society of Edinburgh: Earth Science. 83, 247-257. doi: 10.1017/ S0263593300007938 
Renna, M.R., Tribuzio, R., Tiepolo, M. (2006): Interaction between basic and acid magmas during the latest stages of the post-collisional Variscan evolution: clues from the gabbro-granite association of Ota (Corsica-Sardinia batholith). Lithos 90, 92-110. doi: 10.1016/j. lithos.2006.02.003

Ridolfi, F., Renzulli, A. (2012): Calcic amphiboles in calc-alkaline and alkaline magmas: thermobarometric and chemometric empirical equations valid up to $1.130^{\circ} \mathrm{C}$ and $2.2 \mathrm{GPa}$. Contributions to Mineralogy and Petrology 163, 877-895. doi:10.1007/s00410-011-0704-6. doi: 10.1007/s00410-011-0704-6

Ross, K., Elthon, D. (1997): Cumulus and postcumulus crystallization in the ocean crust: major- and trace-element geochemistry of leg 153 gabbroic rocks. In: J.A. Karson, M. Cannat, D.J. Millet, D. Elthon (eds.), Proceedings of the Ocean Drilling Program, scientific results, TX, pp. 333-350.

Scaillet, B., Evans, B.W. (1999): The 15 June 1991 eruption of Mount Pinatubo. I. Phase equilibria and pre-eruption $\mathrm{P}-\mathrm{T}-\mathrm{fO}_{2}-\mathrm{fH}_{2} \mathrm{O}$ conditions of the dacite magma. Journal of Petrology 40, 381-411. doi: 10.1093/petrology/40.3.381

Scarrow, J.H., Bea, F., Montero, P., Molina, J.F., Vaughan, A.P.M. (2006): A precise late Permian ${ }^{40} \mathrm{Ar} /{ }^{39} \mathrm{Ar}$ age for Central Iberian camptonitic lamprophyres. Geologica Acta 4, 451-459.

Scarrow, J.H., Molina, J.F., Bea, F., Montero, P. (2009): Within-plate calc-alkaline rocks: Insights from alkaline mafic magma-peraluminous crustal melt hybrid appinites of the Central Iberian Variscan continental collision. Lithos 110, 50-64. doi: 10.1016/j.lithos.2008.12.007

Spandler, C., O'Neill, H.S.C. (2010): Diffusion and partition coefficients of minor and trace elements in San Carlos olivine at 1,300C with some geochemical implications. Contributions to Mineralogy and Petrology 159, 791-818. doi: 10.1007/s00410-009-0456-8

Sun, S.S., McDonough, W.F. (1989): Chemical and isotopic systematics of oceanic basalts; implications for mantle composition and processes. In: A.D. Saunders, M.J. Norrey (eds), Magmatism in ocean basins. Blackwell, Geological Society, Special Publication 42, Oxford, pp. 313-345. doi: 10.1144/GSL.SP.1989.042.01.19

Tiepolo, M., Tribuzio, R. (2005): Slab-melting during Alpine orogeny: evidence from mafic cumulates of the Adamello batholith (Central Alps, Italy). Chemical Geology 216, 271-288. doi: 10.1016/j.chemgeo.2004.11.014

Tribuzio, R., Thirlwall, M.F., Messiga, B. (1999a): Petrology, mineral and isotope geochemistry of the Sondalo gabbroic complex (Central Alps, Northern Italy): implications for the origin of post-Variscan magmatism. Contributions to Mineralogy and Petrology 136, 48-62. doi: $10.1007 / \mathrm{s} 004100050523$

Tribuzio, R., Tiepolo, M., Vannucci, R., Bottazzi, P. (1999b): Trace element distribution within olivine-bearing gabbros from the Northern
Apennine ophiolites (Italy): evidence for post-cumulus crystallization in MOR-type gabbroic rocks. Contributions to Mineralogy and Petrology 134, 123-133. doi: 10.1007/s004100050473

Tribuzio, R., Tiepolo, M., Thirlwall, M.F. (2000): Origin of titanian pargasite in gabbroic rocks from the Northern Apennine ophiolites (Italy): insights into the late-magmatic evolution of a MOR-type intrusive sequence. Earth and Planetary Science Letters 176, 281-293. doi: 10.1016/S0012-821X(00)00014-5

Ubide, T., Galé, C., Larrea, P., Arranz, E., Lago, M., Tierz, P. (2014). The relevance of crystal transfer to magma mixing: a case study in composite dykes from the Central Pyrenees. Journal of Petrology 55, 1535-1559. doi: 10.1093/petrology/egu033

Ugidos, J.M., Recio, C. (1993): Origin of cordierite-bearing granites by assimilation in the Central Iberian Zone, Spain. Chemical Geology 103, 27-43. doi: 10.1016/0009-2541(93)90289-U

Villaseca, C., Barbero, L., Rogers, G. (1998): Crustal origin of Hercynian peraluminous granitic batholiths of central Spain: petrological, geochemical and isotopic (Sr,Nd) arguments. Lithos 43, 55-79. doi: 10.1016/S0024-4937(98)00002-4

Villaseca, C., Downes, H., Pin, C., Barbero, L. (1999): Nature and composition of the lower continental crust in central Spain and the granulite-granite linkage: inferences from granulitic xenoliths. Journal of Petrology 40, 1465-1496. doi: 10.1093/petrology/40.10.1465

Villaseca, C., Herreros, V. (2000): A sustained felsic magmatic system: the Hercynian granitic batholith of the Spanish Central System. Transactions of the Royal Society of Edinburgh: Earth Sciences 91, 207-219. doi: 10.1017/S0263593300007380

Villaseca, C., Orejana, D., Pin, C., López García, J.A., Andonaegui, P. (2004): Le magmatisme basique hercynien et post-hercynien du Système Central Espagnol: essai de caractèrisation des sources mantelliques. Comptes Rendus Geosciences 336, 877-888. doi: 10.1016/j. crte.2004.02.008

Villaseca, C., Orejana, D., Paterson, B., Billström, K., Pérez-Soba, C. (2007): Metaluminous pyroxene-bearing granulite xenoliths from the lower continental crust in central Spain: their role in the genesis of Hercynian I-type granites. European Journal of Mineralogy 19, 463 477. doi: 10.1127/0935-1221/2007/0019-1746

Villaseca, C., Orejana, D., Belousova, E.A., Armstrong, R.A., PérezSoba C., Jeffries T.E. (2011): U-Pb isotopic ages and Hf isotope composition of zircons in Variscan gabbros from central Spain: evidence of variable crustal contamination. Mineralogy and Petrology 101, 151-167. doi: 10.1007/s00710-010-0142-6

Zeck, H.P., Wingate, M.T.D., Pooley, G. (2007): Ion microprobe U-Pb zircon geochronology of a late tectonic granitic-gabbroic rock complex within the Hercynian Iberian belt. Geological Magazine 144, 157-177. doi: 10.1017/S0016756806002652

\section{Supplementary material}

(available at http://revistas.ucm.es/index.php/JIGE/)

Supplementary eTable 1.- Major element composition of minerals from Talavera and La Solanilla gabbros (Spanish Central System)

Supplementary eTable 2.- Trace element composition of minerals from Talavera and La Solanilla gabbros (Spanish Central System) 